\title{
A comprehensive model for the kyr and Myr timescales of Earth's axial magnetic dipole field
}

\author{
Matthias Morzfeld $^{1}$ and Bruce A. Buffett ${ }^{2}$ \\ ${ }^{1}$ Department of Mathematics, University of Arizona, 617 N. Santa Rita Ave., P.O. Box 210089, Tucson, AZ 85721, USA \\ ${ }^{2}$ University of California, Department of Earth \& Planetary Science, 307 McCone Hall, Berkeley, CA 94720, USA
}

Correspondence: Matthias Morzfeld (mmo@math.arizona.edu)

Received: 18 September 2018 - Discussion started: 28 September 2018

Revised: 29 May 2019 - Accepted: 12 June 2019 - Published: 8 July 2019

\begin{abstract}
We consider a stochastic differential equation model for Earth's axial magnetic dipole field. Our goal is to estimate the model's parameters using diverse and independent data sources that had previously been treated separately, so that the model is a valid representation of an expanded paleomagnetic record on kyr to Myr timescales. We formulate the estimation problem within the Bayesian framework and define a feature-based posterior distribution that describes probabilities of model parameters given a set of features derived from the data. Numerically, we use Markov chain Monte Carlo (MCMC) to obtain a sample-based representation of the posterior distribution. The Bayesian problem formulation and its MCMC solution allow us to study the model's limitations and remaining posterior uncertainties. Another important aspect of our overall approach is that it reveals inconsistencies between model and data or within the various data sets. Identifying these shortcomings is a first and necessary step towards building more sophisticated models or towards resolving inconsistencies within the data. The stochastic model we derive represents selected aspects of the long-term behavior of the geomagnetic dipole field with limitations and errors that are well defined. We believe that such a model is useful (besides its limitations) for hypothesis testing and give a few examples of how the model can be used in this context.
\end{abstract}

\section{Introduction}

Earth possesses a time-varying magnetic field which is generated by the turbulent flow of liquid metal alloy in the core. The field can be approximated as a dipole with north and south magnetic poles slightly misaligned with the geographic poles. The dipole field changes over a wide range of timescales, from years to millions of years, and these changes are documented by several different sources of data; see, e.g., Hulot et al. (2010). Satellite observations reveal changes in the dipole field over years to decades (Finlay et al., 2016), while changes on timescales of thousands of years are described by paleomagnetic data, including observations of the dipole field derived from archeological artifacts, young volcanics, and lacustrine sediments (Constable et al., 2016). Variations on even longer timescales of millions of years are recorded by marine sediments (Valet et al., 2005; Ziegler et al., 2011) and by magnetic anomalies in the oceanic crust (Ogg, 2012; Cande and Kent, 1995; Lowrie and Kent, 2004). On such long timescales, we can observe the intriguing feature of Earth's axial magnetic dipole field to reverse its polarity (magnetic North Pole becomes the magnetic South Pole and vice versa).

Understanding Earth's dipole field, at any timescale, is difficult because the underlying magnetohydrodynamic problem is highly nonlinear. For example, many numerical simulations are far from Earth-like due to severe computational constraints, and more tractable mean-field models require questionable parameterizations. An alternative approach is to use "low-dimensional models" which aim at providing a simplified but meaningful representation of some aspects of Earth's geodynamo. Several such models have been proposed over the past years. The model of Gissinger (2012), for example, describes the Earth's dipole over millions of years by a set of three ordinary differential equations, one for the dipole, one for the non-dipole field and one for velocity variations at the core. A stochastic model for Earth's dipole over millions 
of years was proposed by Pétrélis et al. (2009). Other models have been derived by Rikitake (1958) and Pétrélis and Fauve (2008).

Following Schmitt et al. (2001) and Hoyng et al. (2001, 2002), we consider a stochastic differential equation (SDE) model for Earth's axial dipole. The basic idea is to model Earth's dipole field analogously to the motion of a particle in a double-well potential. Time variations of the dipole field and dipole reversals then occur as follows. The state of the SDE is within one of the two wells of the double-well potential and is pushed around by noise. The pushes and pulls by the noise process lead to variations of the dipole field around a typical value. Occasionally, however, the noise builds up to push the state over the potential well, which causes a change in its sign. A transition from one well to the other represents a reversal of Earth's dipole. The state of the SDE then remains, for a while, within the opposite well, and the noise leads to time variations of the dipole field around the negative of the typical value. Then, the reverse of this process may occur.

A basic version of this model, which we call the "B13 model" for short, was discussed by Buffett et al. (2013). The drift and diffusion coefficients that define the B13 model are derived from the PADM2M data (Ziegler et al., 2011) which describe variations in the strength of Earth's axial magnetic dipole field over the past 2 Myr. The PADM2M data are derived from marine sediments, which means that the data are smoothed by sedimentation processes; see, e.g., Roberts and Winkhofer (2004). The B13 model, however, does not account directly for the effects of sedimentation. Buffett and Puranam (2017) try to mimic the effects of sedimentation by sending the solution of the SDE through a low-pass filter. With this extension, the B13 model is more suitable for being compared to the data record of Earth's dipole field on a Myr timescale.

A basic assumption of an SDE model is that the noise process within the SDE is uncorrelated in time. This assumption is reasonable when describing the dipole field on the $\mathrm{Myr}$ timescale, but is not valid on a shorter timescale of thousands of years. Buffett and Matsui (2015) derived an extension of the B13 model to extend it to timescales of thousands of years by adding a time-correlated noise process. An extension of $\mathrm{B} 13$ to represent changes in reversal rates over the past $150 \mathrm{Myr}$ is considered by Morzfeld et al. (2018). Its use for predicting the probability of an imminent reversal of Earth's dipole is described by Morzfeld et al. (2017) and by Buffett and Davis (2018). The B13 model is also discussed by Meduri and Wicht (2016), Buffett et al. (2014), and Buffett (2015).

The B13 model and its extensions are constructed with several data sets in mind that document Earth's axial dipole field over the kyr and Myr timescales. The data, however, are not considered simultaneously: the B13 model is based on one data source (paleomagnetic data on the Myr timescale) and some of its modifications are based on other data sources (the shorter record over the past $10 \mathrm{kyr}$ ). Our goal is to con- struct a comprehensive model for Earth's axial dipole field by calibrating the B13 model to several independent data sources simultaneously, including

i. observations of the strength of the dipole over the past $2 \mathrm{Myr}$ as documented by the PADM2M and Sint-2000 data sets (Ziegler et al., 2011; Valet et al., 2005);

ii. observations of the dipole over the past $10 \mathrm{kyr}$ as documented by CALS10k.2 (Constable et al., 2016); and

iii. reversals and reversal rates derived from magnetic anomalies in the oceanic crust (Ogg, 2012).

The approach ultimately leads to a family of SDE models, valid over Myr and kyr timescales, whose parameters are informed by a comprehensive paleomagnetic record composed of the above three sources of data. The results we obtain here are thus markedly different from previous work where data at different timescales are considered separately. We also use our framework to assess the effects of the various data sources on parameter estimates and to discover inconsistencies between model and data.

At the core of our model calibration is the Bayesian paradigm in which uncertainties in data are converted into uncertainties in model parameters. The basic idea is to merge prior information about the model and its parameters, represented by a prior distribution, with new information from data, represented by a likelihood; see, e.g., Reich and Cotter (2015) and Asch et al. (2017). Priors are often assumed to be "uninformative", i.e., that only conservative bounds for all parameters are known, and likelihoods describe point-wise model-data mismatch. Assumed error models in the data can control the effects each data set has on the parameter estimates. Since error models describe what "we do not know", good error models are notoriously difficult to come by. In this context, we discover that the "shortness of the paleomagnetic record," i.e., the limited amount of data available, is the main source of uncertainty. For example, PADM2M or Sint2000 provide a time series of 2000 consecutive "data points" (2 Myr sampled once per kyr). Errors in power spectral densities, computed from such a short time series, dominate the expected errors in these data. Similarly, errors in the reversal rate statistics are likely dominated by the fact that only a small number of reversals, e.g., those that occurred over the past $30 \mathrm{Myr}$, are useful for computing reversal rates. Reliable error models should thus reflect errors due to the shortness of the paleomagnetic record, rather than building error models on assumed errors in the data.

To address these issues we substitute likelihoods based on point-wise mismatch of model and data by a "featurebased" likelihood, as discussed by Maclean et al. (2017) and Morzfeld et al. (2018). Feature-based likelihoods are based on error in "features" extracted from model outputs and data rather than the usual point-wise error. The feature-based approach enables unified contributions from several independent data sources in a well-defined sense even if the various 
Table 1. Time-averaged VADM and VADM standard deviation of PADM2M and Sint-2000.

\begin{tabular}{lrr}
\hline Data source & $\begin{array}{r}\text { Time avg. VADM } \\
\left(10^{22} \mathrm{Am}^{2}\right)\end{array}$ & $\begin{array}{r}\text { SD of VADM } \\
\left(10^{22} \mathrm{Am}^{2}\right)\end{array}$ \\
\hline PADM2M & 5.23 & 1.48 \\
Sint-2000 & 5.81 & 1.84 \\
\hline
\end{tabular}

data may not be entirely self-consistent and further allows us to construct error models that reflect uncertainties induced by the shortness of the paleomagnetic record. In addition, we perform a suite of numerical experiments to check, in hindsight, our a priori assumptions about the error models.

\section{Description of the data}

Variations in the virtual axial dipole moment (VADM) over the past 2 Myr can be derived from stacks of marine sediment. Two different compilations are considered in this study: Sint-2000 (Valet et al., 2005) and PAMD2M (Ziegler et al., 2011). Both of these data sets are sampled every $1 \mathrm{kyr}$ and, thus, provide a time series of 2000 consecutive VADM values. The PADM2M and Sint-2000 data sets are shown in Fig. 1a.

The CALS10k. 2 data set, plotted in Fig. 1b, describes variations of VADM over the past $10 \mathrm{kyr}$ (Constable et al., 2016). The time dependence of CALS10k2 is represented using Bsplines so that the model can be sampled at arbitrary time intervals. We sample CALS10k.2 at an interval of 1 year, although the resolution of CALS10k.2 is nominally 100 years (Constable et al., 2016). Note that we refer to PADM2M, Sint-2000 and CALS10k.2 as "data" because we treat these field reconstructions as such.

Below we use features derived from power spectral densities (PSDs) of the Sint-2000, PADM2M and CALS10k.2 data. The PSDs are computed by the multi-taper spectral estimation technique of Constable and Johnson (2005). A restricted range of frequencies is retained in the estimation to account for data resolution and other complications (see below). We show the resulting PSDs of the three data sets in Fig. 1c. During parameter estimation we further make use of the time-averaged VADM and the standard deviation of VADM over time of the Sint-2000 and PADM2M data sets listed in Table 1.

Lastly, we make use of reversal rates of the Earth's dipole computed from the geomagnetic polarity timescale (Cande and Kent, 1995; Lowrie and Kent, 2004; Ogg, 2012). Using the chronology of Ogg (2012), we compute reversal rates for $5 \mathrm{Myr}$ intervals from today up to $30 \mathrm{Myr}$ ago. That is, we compute the reversal rates for the intervals $0-5,5-10, \ldots$, 25-30 Myr. This leads to the average reversal rate and standard deviation listed in Table 2.
Table 2. Average reversal rate and standard deviation computed over the past $30 \mathrm{Myr}$ using the chronology of Ogg (2012).

\begin{tabular}{lrr}
\hline Interval length & $\begin{array}{r}\text { Average reversal rate } \\
\text { (reversals per Myr) }\end{array}$ & $\begin{array}{r}\text { SD } \\
\text { (reversals per Myr) }\end{array}$ \\
\hline $5 \mathrm{Myr}$ & 4.23 & 1.01 \\
$10 \mathrm{Myr}$ & 4.23 & 0.49 \\
\hline
\end{tabular}

Increasing the interval to $10 \mathrm{Myr}$ leads to the same mean but decreases the standard deviation (see Table 2).

Note that the various data are not all consistent. For example, visual inspection of VADM (Fig. 1) as well as comparison of the time average and standard deviation (Table 1) indicate that the PADM2M and Sint-2000 data sets report different VADMs. These differences can be attributed, at least in part, to differences in the calibration of the marine sediment measurements and to differences in the way the measurements are stacked to recover the dipole component of the field. There are also notable differences between the PSDs from CALS10k.2 and those from the lower-resolution data sets (SINT-2000 and PADM2M) at the overlapping frequencies. Dating uncertainties, smoothing due to sedimentary processes and the finite duration of the records all contribute to these discrepancies. We do not attempt to identify the source of these discrepancies. Instead, we seek to recover parameter values for a stochastic model by combining a feature-based approach with realistic estimates of the data uncertainty (see Sect. 4).

We further note that the amount of data is rather limited: we have $2 \mathrm{Myr}$ of VADM sampled at 1 per kyr and $10 \mathrm{kyr}$ of high-frequency VADM, and use a $30 \mathrm{Myr}$ record to compute reversal rates. The limited amount of data directly affects how the accuracy of the data should be interpreted. As an example, the mean and standard deviation of the reversal rate, based on a $30 \mathrm{Myr}$ record, may not be accurate; errors in the PSDs of PADM2M, Sint-2000 or CALS10k.2 are dominated by the fact that these are computed from relatively short time series. We address these issues by using the feature-based approach that allows us to build error models that reflect uncertainties due to the shortness of the paleomagnetic record. We further perform extensive numerical tests that allow us to check, in hindsight, the validity of our assumptions about errors (see Sect. 6).

\section{Description of the model}

Our models for variations in the dipole moment on Myr and kyr timescales are based on a scalar SDE:

$\mathrm{d} x=v(x) \mathrm{d} t+\sqrt{2 D(x)} \mathrm{d} W$,

where $t$ is time and where $x$ represents the VADM and polarity of the dipole; see, e.g., Schmitt et al. (2001), 

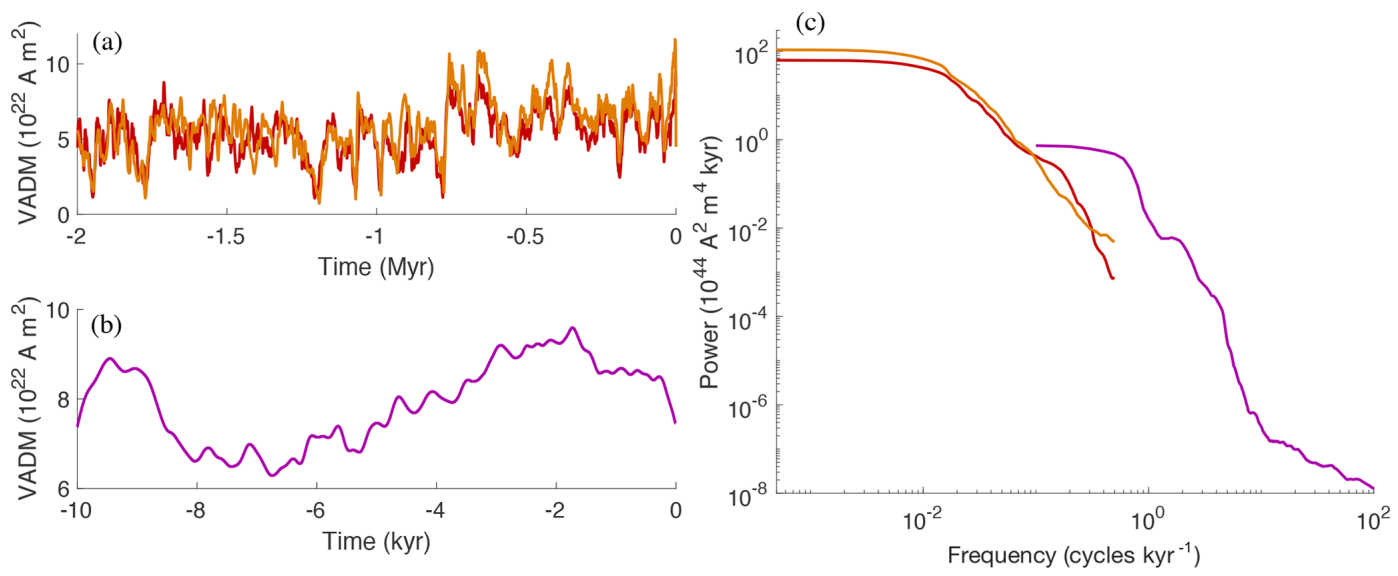

Figure 1. Data used in this paper. (a) Sint-2000 (orange) and PADM2M (red): VADM as a function of time over the past 2 Myr. (b) CALS10k.2: VADM as a function of time over the past $10 \mathrm{kyr}$. (c) Power spectral densities of the data in (a) and (b), computed by the multi-taper spectral estimation technique of Constable and Johnson (2005). Orange: Sint-2000. Red: PADM2M. Purple: CALS10k.2.

Hoyng et al. (2001, 2002), and Buffett et al. (2013). A negative sign of $x(t)$ corresponds to the current polarity, and a positive sign means reversed polarity. $W$ is Brownian motion, a stochastic process with the following properties: $W(0)=0$, $W(t)-W(t+\Delta T) \sim \mathcal{N}(0, \Delta t)$, and $W(t)$ is almost surely continuous for all $t \geq 0$; see, e.g., Chorin and Hald (2013). Here and below, $\mathcal{N}\left(\mu, \sigma^{2}\right)$ denotes a Gaussian random variable with mean $\mu$, standard deviation $\sigma$ and variance $\sigma^{2}$. Throughout this paper, we assume that the diffusion, $D(x)$, is constant, i.e., that $D(x)=D$. Modest variations in $D$ have been reported on the basis of geodynamo simulations (Buffett and Matsui, 2015; Meduri and Wicht, 2016). Representative variations in $D$, however, have a small influence on the statistical properties of solutions of the SDE (Eq. 1); see Buffett and Matsui (2015).

The function $v$ is called the "drift" and is derived from a double-well potential, $U^{\prime}(x)=-v(x)$. Here, we consider drift coefficients of the form

$v(x)=\gamma \frac{x}{\bar{x}} \cdot\left\{\begin{array}{ll}(\bar{x}-x), & \text { if } x \geq 0 \\ (x+\bar{x}), & \text { if } x<0\end{array}\right.$,

where $\bar{x}$ and $\gamma$ are parameters. The parameter $\bar{x}$ defines where the drift coefficient vanishes and also corresponds to the time average of the associated linear model:

$\mathrm{d} x^{l}=-\gamma\left(x^{l}-\bar{x}\right) \mathrm{d} t+\sqrt{D} \mathrm{~d} W$,

which is obtained by Taylor expanding $v(x)$ at $\bar{x}$. It is now clear that the parameter $\gamma$ defines a relaxation time.

Nominal values of the parameters $\bar{x}, \gamma$ and $D$ are listed in Table 3.

With the nominal values, the model exhibits "dipole reversals", which are represented by a change in the sign of $x$. This is the "basic" B13 model.

For computations, we discretize the SDE using a fourthorder Runge-Kutta (RK4) method for the drift and an EulerMaruyama method for the diffusion. This results in the
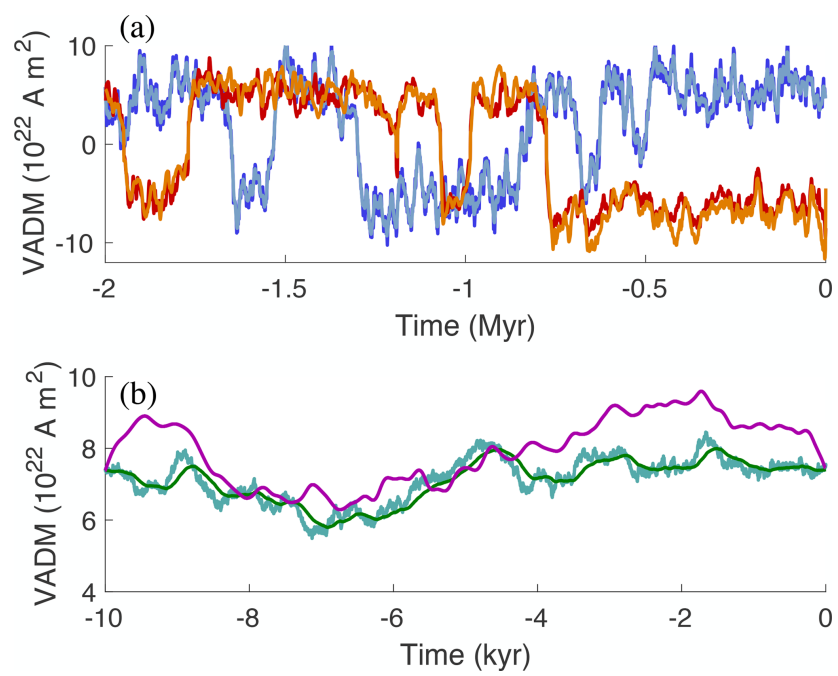

Figure 2. Simulations with nominal parameter values and data on the Myr and kyr scales. (a) VADM as a function of time on the Myr timescale. The output of the Myr model, $x_{j}^{\mathrm{Myr}}$, is shown in dark blue (often hidden). The smoothed output, $x_{j}^{\mathrm{Myr}, \mathrm{s}}$, is shown in light blue. The signed Sint-2000 and PADM2M are shown in orange and red with signs (reversal timings) taken from Cande and Kent (1995). (b) VADM as a function of time on the kyr timescale. The output of the kyr model with uncorrelated noise is shown in turquoise. The output of the kyr model with correlated noise is shown in green. VADM of CALS10k.2 is shown in purple.

discrete-time B13 model

$x_{k}=f\left(x_{k-1}, \Delta t\right)+\sqrt{2 D \Delta t} w_{k}, w_{k} \sim \mathcal{N}(0,1)$, iid,

where $\Delta t$ is the time step, where $\sqrt{\Delta t} w_{k}$ is the discretization of Brownian motion $W$ in Eq. (1) and where $f\left(x_{k-1}, \Delta t\right)$ is the RK4 step. Here, iid stands for "independent and identically distributed"; i.e., each random variable 
Table 3. Nominal parameter values and parameter bounds.

\begin{tabular}{lrrrrr}
\hline & $\bar{x}$ & $D$ & $\gamma$ & $T_{\mathrm{S}}$ & $\begin{array}{r}a \\
\left(10^{22} \mathrm{~A} \mathrm{~m}^{2}\right)\end{array}$ \\
& $\left(10^{44} \mathrm{~A}^{2} \mathrm{~m}^{4} \mathrm{kyr}^{-1}\right)$ & $\left(\mathrm{kyr}^{-1}\right)$ & $(\mathrm{kyr})$ & $\left(\mathrm{kyr}^{-1}\right)$ \\
\hline Nominal value & 5.23 & 0.3403 & 0.075 & 2.5 & 5 \\
Lower bound & 0 & 0.0615 & 0.0205 & 1 & 5 \\
Upper bound & 10 & 2.1 & 0.7 & 5 & 40 \\
\hline
\end{tabular}

$w_{k}$, for $k>0$, has the same Gaussian probability distribution, $\mathcal{N}(0,1)$, and $w_{i}$ and $w_{j}$ are independent for all $i \neq j$. We distinguish between variations in the Earth's dipole over kyr to Myr timescales and, for that reason, present modifications of the basic B13 model (Eq. 4).

\subsection{Models for the Myr amd kyr timescales}

For simulations over Myr timescales we chose a time step $\Delta t=1 \mathrm{kyr}$, corresponding to the sampling time of the Sint2000 and PADM2M data. On a Myr timescale, the primary sources of paleomagnetic data in Sint-2000 and PADM2M are affected by gradual acquisition of magnetization due to sedimentation processes, which amount to an averaging over a (short) time interval; see, e.g., Roberts and Winkhofer (2004). We follow Buffett and Puranam (2017) and include the smoothing effects of sedimentation in the model by convolving the solution of Eq. (4) by a Gaussian filter

$g(t)=\sqrt{\frac{6}{\pi T_{\mathrm{S}}^{2}}} \cdot \exp \left(-\frac{6 t^{2}}{T_{\mathrm{s}}^{2}}\right)$,

where $T_{\mathrm{S}}$ defines the duration of smoothing, i.e., the width of a time window over which we average. The nominal value for $T_{\mathrm{S}}$ is given in Table 3 . The result is a smoothed Myr model whose state is denoted by $x^{\mathrm{Myr}, \mathrm{s}}$. Simulations with the "Myr model" and the nominal parameters of Table 3 are shown in Fig. 2a, where we plot the model output $x_{j}^{\text {Myr }}$ in dark blue and the smoothed model output, $x_{j}^{\mathrm{Myr}, \mathrm{s}}$, in a lighter blue over a period of $2 \mathrm{Myr}$.

On a Myr scale, the assumption that the noise is uncorrelated in time is reasonable because one focuses on low frequencies and large sample intervals of the dipole, as in Sint2000 and PADM2M, whose sampling interval is $1 / \mathrm{kyr}$. On a shorter timescale, as in CALS10k.2, this assumption is not valid and a correlated noise is more appropriate (Buffett and Matsui, 2015). Computationally, this means that we swap the uncorrelated, iid, noise in Eq. (4) for a noise that has a short but finite correlation time. This can be done by "filtering" Brownian motion. The resulting discrete time model for the kyr timescale is

$y_{k}=(1-a \Delta t) y_{k-1}+\sqrt{2 a \Delta t} w_{k}, w_{k} \sim \mathcal{N}(0,1)$, iid

$x_{k}=f\left(x_{k-1}, \Delta t\right)+\sqrt{D a} \Delta t y_{k}$,

where $a$ is the model parameter that defines the correlation time $T_{\mathrm{c}}=1 / a$ of the noise and $\Delta t=1 \mathrm{yr}$. A $10 \mathrm{kyr}$ simula- tion of the kyr models with uncorrelated and correlated noise using the nominal parameters of Table 3 is shown in Fig. $2 b$ along with the CALS10k.2 data.

\subsection{Approximate power spectral densities}

Accurate computation of the PSDs from the time-domain solution of the B13 model requires extremely long simulations. For example, the PSDs of two (independent) 1 billion year simulations with the Myr model are still surprisingly different. In fact, errors that arise due to "short" simulations substantially outweigh errors due to linearization. Recall that the PSD of the linear model (Eq. 3) is easily calculated to be

$\hat{x}^{l}(f)=\frac{2 D}{\gamma^{2}+4 \pi^{2} f^{2}}$,

where $f$ is the frequency (in $1 / \mathrm{kyr}$ ). Since the Fourier transform of the Gaussian filter is known analytically, the PSD of the smoothed linear model is also easy to calculate:

$\hat{x}^{l, s}(f)=\frac{2 D}{\gamma^{2}+4 \pi^{2} f^{2}} \cdot \exp \left(-\frac{4 \pi^{2} f^{2} T_{\mathrm{s}}^{2}}{12}\right)$.

Similarly, an analytic expression for the PSD of the kyr model with correlated noise in Eqs. (6)-(7) can be obtained by taking the limit of continuous time $(\Delta t \rightarrow 0)$ :

$\hat{x}^{l, \mathrm{kyr}}(f)=\frac{2 D}{\gamma^{2}+4 \pi^{2} f^{2}} \cdot \frac{a^{2}}{a^{2}+4 \pi^{2} f^{2}}$.

Here, the first term is as in Eq. (8) and the second term appears because of the correlated noise.

Figure 3 illustrates a comparison of the PSDs obtained from simulations of the nonlinear models and their linear approximations.

Specifically, the PSDs of the (smoothed) Myr scale nonlinear model, computed from a $50 \mathrm{Myr}$ simulation, are shown in comparison to the approximate PSDs in Eqs. (8)-(9). Note that the PSD of the smoothed model output, $x^{\mathrm{Myr}, \mathrm{s}}$, taking into account sedimentation processes, rolls off quicker than the PSD of $x_{j}^{\mathrm{Myr}}$. For that reason, the PSD of the smoothed model seems to fit the PSDs of the Sint-2000 and PADM2M data "better"; i.e., we observe a similarly quick roll-off at high frequencies in model and data; see also Buffett and $\mathrm{Pu}-$ ranam (2017). The PSD of the kyr model with correlated noise, computed from a $10 \mathrm{kyr}$ simulation, is also shown in 
(a)

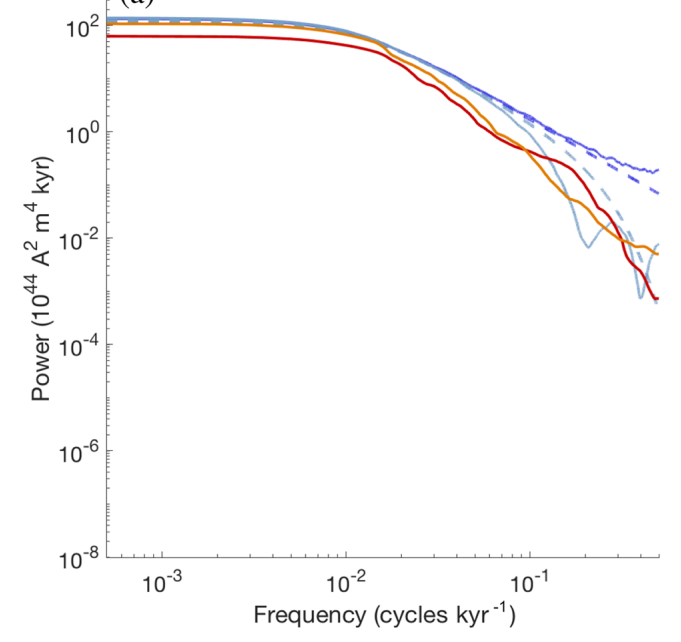

(b)

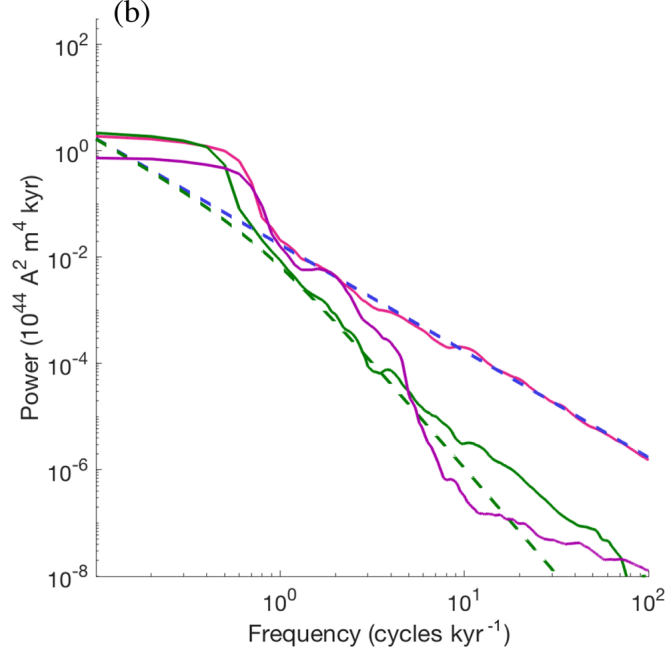

Figure 3. Power spectral densities of the model with nominal parameter values. (a) Myr model: a PSD of a 50 Myr simulation with the Myr model is shown as a solid dark blue line. The corresponding theoretical PSD of the linear model is shown as a dashed dark blue line. A PSD of a $50 \mathrm{Myr}$ simulation with the Myr model and high-frequency roll-off is shown as a solid light blue line. The corresponding theoretical PSD of the linear model is shown as a dashed light blue line. The PDSs of Sint-2000 and PADM2M are shown in orange and red. (b) kyr model: a PSD of a $10 \mathrm{kyr}$ simulation of the kyr model with uncorrelated noise is shown as a solid pink line. The corresponding theoretical spectrum of the linear model is shown as a dashed blue line. A PSD of a $10 \mathrm{kyr}$ simulation of the kyr model with correlated noise is shown as a solid green line. The corresponding theoretical spectrum is shown as a dashed green line. The PDSs of CALS10k.2 is shown in purple. All PSDs are computed by the multi-taper spectral estimation technique of Constable and Johnson (2005).

Fig. 3 in comparison with the linear PSD in Eq. (10). The good agreement between the theoretical spectra of the linear models and the spectra of the nonlinear models justifies the use of the linear approximation. We have further noted in numerical experiments that the agreement between the nonlinear and linear spectra increases with increasing simulation time; however, a "perfect" match requires extremely long simulations of the nonlinear model (hundreds of billions of years). The approximate PSDs, based on the linear models, will prove useful in the construction of likelihoods in Sect. 4.2.

In addition to a good match of the PSDs of the nonlinear and linear models, we note that the PSDs of the model match, at least to some extent, the PSDs of the data (Sint-2000, PADM2M and CALS10k.2). This means that our choice for the nominal values is "reasonable" because this choice leads to a reasonable fit to the data. The goal of using a Bayesian approach to parameter estimation, described in Sect. 4, is to improve this fit and to equip the (nominal) parameter values with an error estimate, i.e., to define and compute a distribution over the model parameters.

\subsection{Approximate reversal rate, VADM time average and VADM standard deviation}

The nonlinear SDE model (Eq. 1) and its discretization (Eq. 4) exhibit reversals, i.e., a change in the sign of $x$. Moreover, the overall "power", i.e., the area under the PSD curve, is given by the standard deviation of the absolute value of $x(t)$ over time. Another important quantity of interest is the time-averaged value of the absolute value of $x(t)$, which describes the average strength of the dipole field. In principle, these quantities (reversal rate, time average and standard deviation) can be computed from simulations of the Myr and kyr models in the time domain. Similarly to what we found in the context of PSD computations and approximations, we find that estimates of the reversal rate, time average and standard deviation are subject to large errors unless the simulation time is very long (hundreds of millions of years). Using the linear model and Kramer's formula, however, one can approximate the time average, reversal rate and standard deviation without simulating the nonlinear model (see below). Computing the approximate values is instantaneous (evaluation of simple formulas) and the approximations are comparable to what we obtain from very long simulations with the nonlinear model. As is the case with the PSD approximations based on linear models, the below approximations of the reversal rate, time average and standard deviation will prove useful for formulating likelihoods in Sect. 4.2.

Specifically, the parameter $\bar{x}$ defines the time average of the linear model (Eq. 3), and it also defines where the drift term (Eq. 2) vanishes. These values coincide quite closely with the time average of the nonlinear model which suggests the approximation

$E(x) \approx \bar{x} \times 10^{22} \mathrm{~A} \mathrm{~m}^{2}$. 
The reversal rate can be approximated by Kramer's formula (Buffett and Puranam, 2017; Risken, 1996):

$r \approx \frac{\gamma}{2 \pi} \cdot \exp \left(-\frac{\gamma \bar{x}^{2}}{6 D}\right) \times 10^{3} \mathrm{Myr}^{-1}$.

The standard deviation is the square root of the area under the PSD. Using the linear model that incorporates the effects of smoothing (due to sedimentation), one can approximate the standard deviation by computing the integral of the PSD in Eq. (9):

$\sigma \approx\left(\frac{D}{\gamma} \exp \left(\frac{\left(\gamma T_{\mathrm{S}}\right)^{2}}{12}\right) \operatorname{erfc}\left(\frac{\gamma T_{\mathrm{s}}}{2 \sqrt{3}}\right)\right)^{1 / 2} \times 10^{22} \mathrm{Am}^{2}$,

where $\operatorname{erfc}(\cdot)$ is the (Gaussian) error function. Without incorporating the smoothing, the standard deviation based on the linear model would be the integral of the PDS in Eq. (8), which is $\sigma \approx \sqrt{D / \gamma}$. The exponential and error function terms in Eq. (13) can thus be interpreted as a correction factor that accounts for the effects of sedimentation. It is easy to check that this correction factor is always smaller than 1, i.e., that the (approximate) standard deviation accounting for sedimentation effects is smaller than the (approximate) standard deviation that does not account for these effects.

For the nominal parameter values in Table 3 we calculate a time average of $\bar{x}=5.23 \times 10^{22} \mathrm{~A} \mathrm{~m}^{2}$, a standard deviation of $\sigma \approx 2.07 \times 10^{22} \mathrm{~A} \mathrm{~m}^{2}$ and a reversal rate of $r \approx 4.37 \mathrm{Myr}^{-1}$. These should be compared to the corresponding values of PADM2M and Sint-2000 in Table 1 and to the reversal rate from the geomagnetic polarity timescale in Table 2. Similar to what we observed of the model-data fit in terms of (approximate) PSDs, we find that the nominal parameter values lead to a "reasonable" fit of the model's reversal rate, time average and standard deviation. The Bayesian parameter estimation in Sect. 4 will improve this fit and lead to a better understanding of model uncertainties.

\subsection{Parameter bounds}

The Bayesian parameter estimation, described in Sect. 4, makes use of "prior" information about the model parameters. We formulate prior information in terms of parameter bounds and construct uniform prior distributions with these bounds. The parameter bounds we use are quite wide; i.e., the upper bounds are probably too large and the lower bounds are probably too small, but this is not critical for our purposes, as we explain in more detail in Sect. 4.

The parameter $\gamma$ is defined by the inverse of the dipole decay time (Buffett et al., 2013). An upper bound on the dipole decay time $\tau_{\text {dec }}$ is given by the slowest decay mode $\tau_{\mathrm{dec}} \leq R^{2} /\left(\pi^{2} \eta\right)$, where $R$ is the radius of the Earth and $\eta=$ $0.8 \mathrm{~m}^{2} \mathrm{~s}^{-1}$ is the magnetic diffusivity. Thus, $\tau_{\mathrm{dec}} \leq 48.6 \mathrm{kyr}$, which means that $\gamma \geq 0.0205 \mathrm{kyr}^{-1}$. This is a fairly strict lower bound because the dipole may relax on timescales shorter than the slowest decay mode, and a recent theoretical calculation (Pourovskii et al., 2017) suggests that the magnetic diffusivity may be slightly larger than $0.8 \mathrm{~m}^{2} \mathrm{~s}^{-1}$. Both of these changes would cause the lower bound for $\gamma$ to increase. To obtain an upper bound for $\gamma$, we note that if $\gamma$ is large, the magnetic decay is short, which means that it becomes increasingly difficult for convection in the core to maintain the magnetic field. The ratio of dipole decay time $\tau_{\text {dec }}$ to advection time $\tau_{\mathrm{adv}}=L / V$, where $L=2259 \mathrm{~km}$ is the width of the fluid shell and $V=0.5 \mathrm{~mm} \mathrm{~s}^{-1}$, needs to be $10: 1$ or (much) larger. This leads to the upper bound $\gamma \leq 0.7 \mathrm{kyr}^{-1}$.

Bounds for the parameter $D$ can be found by considering the linear Myr timescale model in Eq. (3), which suggests that the variance of the dipole moment is $\operatorname{var}(x)=$ $D / \gamma$; see also Buffett et al. (2013). Thus, we may require that $D \sim \operatorname{var}(x) \gamma$. The average of the variance of Sint$2000\left(\operatorname{var}(x)=3.37 \times 10^{44} \mathrm{~A}^{2} \mathrm{~m}^{4}\right)$ and PADM2M $(\operatorname{var}(x)=$ $\left.2.19 \times 10^{44} \mathrm{~A}^{2} \mathrm{~m}^{4}\right)$ is $\operatorname{var}(x) \approx 2.78 \times 10^{44} \mathrm{~A}^{2} \mathrm{~m}^{4}$. We use the rounded-up value $\operatorname{var}(x) \approx 3 \times 10^{44} \mathrm{~A}^{2} \mathrm{~m}^{4}$ and, together with the lower and upper bounds on $\gamma$, this leads to the lower and upper bounds $0.062 \times 10^{44} \leq D \leq 2.1 \times 10^{44} \mathrm{~A}^{2} \mathrm{~m}^{4} \mathrm{kyr}^{-1}$.

The smoothing time, $T_{\mathrm{s}}$, due to sedimentation and the correlation parameter for the noise, $a$, define the roll-off frequency of the power spectra for the Myr and kyr models, respectively. We assume that $T_{\mathrm{S}}$ is within the interval $[1,5] \mathrm{kyr}$ and that the correlation time $a^{-1}$ is within $[0.025,0.2] \mathrm{kyr}$ (i.e., $a$ within $[5,40] \mathrm{kyr}^{-1}$ ). These choices enforce that $T_{\mathrm{S}}$ controls roll-off at lower frequencies (Myr model) and $a$ controls the roll-off at higher frequencies (kyr model). Bounds for the parameter $\bar{x}$ are not easy to come by and we assume wide bounds, $\bar{x} \in[0,10] \times 10^{22} \mathrm{Am}^{2}$. Here, $\bar{x}=0$ is the lowest lower bound we can think of since the average value of the field is always normalized to be positive. The value of the upper bound of $\bar{x} \leq 10$ is chosen to be excessively large - the average field strength over the last $2 \mathrm{Myr}$ is $\bar{x} \approx 5$. Lower and upper bounds for all five model parameters are summarized in Table 3.

\section{Formulation of the Bayesian parameter estimation problem and numerical solution}

The family of models, describing kyr and Myr timescales and accounting for sedimentation processes and correlations in the noise process, has five unknown parameters, $\bar{x}, D, \gamma, T_{\mathrm{s}}$, and $a$. We summarize the unknown parameters in a "parameter vector" $\theta=\left(\bar{x}, D, \gamma, T_{\mathrm{s}}, a\right)^{T}$. Our goal is to estimate the parameter vector $\theta$ using a Bayesian approach, i.e., to sharpen prior knowledge about the parameters by using the data described in Sect. 2. This is done by expressing prior information about the parameters in a prior probability distribution $p_{0}(\theta)$ and by defining a likelihood $p_{l}(y \mid \theta), y$ being shorthand notation for the data of Sect. 2. The prior distribution describes information we have about the parameters independently of the data. The likelihood describes the probability of the data given the parameters $\theta$ and, therefore, con- 
nects model output and data. The prior and likelihood define the posterior distribution

$p(\theta \mid y) \propto p_{0}(\theta) p_{l}(y \mid \theta)$.

The posterior distribution combines the prior information with the information we extract from the data. In particular, we can estimate parameters based on the posterior distribution. For example, we can compute the posterior mean and posterior standard deviation for the various parameters, and we can also compute correlations between the parameters. The posterior distribution contains all information we have about the model parameters, given prior knowledge and information extracted from the data. Thus, the SDE model with random parameters, whose distribution is the posterior distribution, represents a comprehensive model of the Earth's dipole in view of the data we use.

On the other hand, the posterior distribution depends on several assumptions: since we define the prior and likelihood, we also implicitly define the posterior distribution. In particular, formulations of the likelihood require that one be able to describe anticipated errors in the data as well as anticipated model error. Such error models are difficult to come by in general, but even more so when the amount of data is limited. We address this issue by first formulating "reasonable" error models, followed by a set of numerical tests that confirm (or disprove) our choices of error models (see Sect. 6). In our formulation of error models, we focus on errors that arise due to the shortness of the paleomagnetic record because these errors dominate.

We solve the Bayesian parameter estimation problem numerically by using a Markov chain Monte Carlo (MCMC) method. An MCMC method generates a (Markov) chain of parameter values whose stationary distribution is the posterior distribution. The chain is constructed by proposing a new parameter vector and then accepting or rejecting this proposal with a specified probability that takes the posterior probability of the proposed parameter vector into account. A numerical solution via MCMC thus requires that the likelihood be evaluated for every proposed parameter vector. Below we formulate a likelihood that involves computing the PSDs of the Myr and kyr models, as well as reversal rates, time averages and standard deviations. As explained in Sect. 3, obtaining these quantities from simulations with the nonlinear models requires extremely long simulations. Long simulations, however, require more substantial computations. This perhaps would not be an issue if we were to compute the PDSs, reversal rate and other quantities once, but the MCMC approach we take requires repeated computing. For example, we consider Markov chains of length $10^{6}$, which requires $10^{6}$ computations of PSDs, reversal rates, etc. Moreover, we will repeat these computations in a variety of settings to assess the validity of our error models (see Sect. 6). To keep the computations feasible (fast), we thus decided to use the approximations of the PSDs, reversal rate, time average and standard deviation, based on the linear models (see Sect. 3), to define the likelihood. Evaluation of the likelihood is then instantaneous because simulations with the nonlinear models are replaced by formulas that are simple to evaluate. Using the approximation is further justified by the fact that the approximate PDSs, reversal rates, time averages and standard are comparable to what we obtain from very long simulations with the nonlinear model.

\subsection{Prior distribution}

The prior distribution describes knowledge about the model parameters we have before we consider the data. In Sect. 3.4, we discussed lower and upper bounds for the model parameters and we use these bounds to construct the prior distribution. This can be achieved by assuming a uniform prior over a five-dimensional hyper-cube whose corners are defined by the parameter bounds in Table 1. Note that the bounds we derived in Sect. 3.4 are fairly wide. Wide bounds are preferable for our purposes, because wide bounds implement minimal prior knowledge about the parameters. With such "uninformative priors", the posterior distribution, which contains information from the data, reveals how well the parameter values are constrained by data. More specifically, if the uniform prior distribution is morphed into a posterior distribution that describes a well-defined "bump" of posterior probability mass in parameter space, then the model parameters are constrained by the data (to be within the bump of posterior probability "mass"). If the posterior distribution is nearly equal to the prior distribution, then the data have nearly no effect on the parameter estimates and, therefore, the data do not constrain the parameters.

\subsection{Feature-based likelihoods}

We wish to use a collection of paleomagnetic observations to calibrate and constrain all five model parameters. For this purpose, we use the data sets Sint-2000, PADM2M and CALS10k.2, as well as information about the reversal rate based on the geomagnetic polarity timescale (see Sect. 2). The various data sets are not consistent and, for example, Sint-2000 and PADM2M report different VADM values at the same time instant (see Fig. 1). Likelihoods that are defined in terms of a point-wise mismatch of model and data balance the effects of each data set via (assumed) error covariances: the data set with smaller error covariances has a stronger effect on the parameter estimates. Accurate error models, however, are hard to come by. For this reason, we use an alternative approach called "feature-based data assimilation" (see Morzfeld et al., 2018; Maclean et al., 2017). The idea is to extract "features" from the data and to subsequently define likelihoods that are based on the mismatch of the features derived from the data and the model. Below, we formulate features that account for discrepancies across the various data sources and derive error models for the features. The error models are built to reflect uncertainties that arise due 
to the shortness of the paleomagnetic record. The resulting feature-based posterior distribution describes the probability of model parameters in view of the features. Thus, model parameters with a large feature-based posterior probability lead to model features that are comparable to the features derived from the data, within the assumed uncertainties due to the shortness of the paleomagnetic record.

Specifically, we define likelihoods based on features derived from PSDs of the Sint-2000, PADM2M and CALS10k.2 data sets, as well as the reversal rate, timeaveraged VADM and VADM standard deviation. The overall likelihood consists of three factors:

i. one factor corresponds to the contributions from the reversal rate, time-averaged VADM and VADM standard deviation data, which we summarize as "time-domain data" from now on for brevity;

ii. one factor describes the contributions from data at low frequencies of $10^{-4}-0.5$ cycles per kyr (PADM2M and Sint-2000); and

iii. one factor describes the contributions of data at high frequencies of 0.9-9.9 cycles per kyr (CALS10k.2).

In the Bayesian approach, and assuming that errors are independent, this means that the likelihood $p_{l}(y \mid \theta)$ in Eq. (14) can be written as the product of three terms:

$p_{l}(y \mid \theta) \propto p_{l, \mathrm{td}}(y \mid \theta) p_{l, \mathrm{lf}}(y \mid \theta) p_{l, \mathrm{hf}}(y \mid \theta)$,

where $p_{l, \mathrm{td}}(y \mid \theta), p_{l, \text { lf }}(y \mid \theta)$ and $p_{l, \mathrm{hf}}(y \mid \theta)$ represent the contributions from the time-domain data (reversal rate, timeaveraged VADM and VADM standard deviation), the low frequencies and the high frequencies; recall that $y$ is shorthand notation for all the data we use. We now describe how each component of the overall likelihood is constructed.

\subsubsection{Reversal rates, time-averaged VADM and VADM standard deviation}

We define the likelihood component of the time-domain data based on the equations

$y_{\mathrm{rr}}=h_{\mathrm{rr}}(\theta)+\varepsilon_{\mathrm{rr}}$,

$y_{\bar{x}}=h_{\bar{x}}(\theta)+\varepsilon_{\bar{x}}$,

$y_{\sigma}=h_{\sigma}(\theta)+\varepsilon_{\sigma}$,

where $y_{\mathrm{rr}}, y_{\bar{x}}$, and $y_{\sigma}$ are features derived from the timedomain data, and $h_{\mathrm{rr}}(\theta), h_{\bar{x}}(\theta)$ and $h_{\sigma}(\theta)$ are functions that connect the model parameters to the features, based on the approximations described in Sect. 3.3. In addition to assuming independent errors, we further assume that all errors are Gaussian, i.e., that $\varepsilon_{\mathrm{rr}}, \varepsilon_{\bar{x}}$ and $\varepsilon_{\sigma}$ are independent Gaussian error models with mean zero and variances $\sigma_{\mathrm{rr}}^{2}, \sigma_{\bar{x}}^{2}$, and $\sigma_{\sigma}^{2}$. A Gaussian assumption of errors is widely used. Note that errors are notoriously difficult to model, which also makes it difficult to motivate and justify a particular statistical description. The wide use of Gaussian errors can be explained, at least in part, by Gaussian errors being numerically easy to deal with. We use Gaussian errors for these reasons, but the overall approach we describe can also be extended to be used along with other assumptions about errors if these were available.

Taken all together, the likelihood term $p_{l, \mathrm{td}}(y \mid \theta)$ in Eq. (15) is then given by the product of the three likelihoods defined by Eqs. (16), (17) and (18):

$$
\begin{aligned}
p_{l, \mathrm{td}}(y \mid \theta) & \propto \exp \left(-\frac{1}{2}\left(\left(\frac{y_{\mathrm{rr}}-h_{\mathrm{rr}}(\theta)}{\sigma_{\mathrm{rr}}}\right)^{2}+\left(\frac{y_{\bar{x}}-h_{\bar{x}}(\theta)}{\sigma_{\bar{x}}}\right)^{2}\right.\right. \\
& \left.\left.+\left(\frac{y_{\sigma}-h_{\sigma}(\theta)}{\sigma_{\sigma}}\right)^{2}\right)\right) .
\end{aligned}
$$

The reversal rate feature is simply the average reversal rate we computed from the chronology of Ogg (2012) (see Sect. 2), i.e., $y_{\mathrm{rr}}=4.23$ reversals per Myr. The function $h_{\mathrm{rr}}(\theta)$ is based on the approximation using Kramer's formula in Eq. (20):

$$
\begin{aligned}
h_{\mathrm{rr}}(\theta) & =\frac{\gamma}{2 \pi} \exp \left(-\frac{\gamma \bar{x}^{2}}{6 D}\right) \\
& \times 10^{3} \text { reversals per Myr. }
\end{aligned}
$$

The time-average feature is the mean of the time averages of PADM2M and Sint-2000: $y_{\bar{x}}=5.56 \times 10^{22} \mathrm{Am}^{2}$. The function $h_{\bar{x}}(\theta)$ is based on the linear approximation discussed in Sect. 3.3, i.e., $h_{\bar{x}}(\theta)=\bar{x}$. The feature for the VADM standard deviation is the average of the VADM standard deviations of PADM2M and Sint-2000: $y_{\sigma}=1.66 \times 10^{22} \mathrm{Am}^{2}$. The function $h_{\sigma}(\theta)$ uses the linear approximation of the standard deviation (Eq. 13):

$$
\begin{aligned}
h_{\sigma}(\theta) & =\left(\frac{D}{\gamma} \exp \left(\frac{\left(\gamma T_{\mathrm{S}}\right)^{2}}{12}\right) \operatorname{erfc}\left(\gamma T_{\mathrm{s}} / 2 / \sqrt{(3)}\right)\right)^{1 / 2} \\
& \times 10^{22} \mathrm{Am}^{2} \mathrm{kyr}^{2} .
\end{aligned}
$$

Candidate values for these error variances are as follows The error variance of the reversal rate, $\sigma_{\mathrm{rr}}^{2}$, can be based on the standard deviations we computed from the Ogg (2012) chronology in Table 2. Thus, we might use the standard deviation of the $10 \mathrm{Myr}$ average and take $\sigma_{\mathrm{rr}}=0.5$. One can also use the model with nominal parameter values (see Table 3 ) to compute candidate values of the standard deviation $\sigma_{\mathrm{rr}}$. We perform 1000 independent $10 \mathrm{Myr}$ simulations and, for each simulation, determine the reversal rate. The standard deviation of the reversal rate based on these simulations is 0.69 reversals per Myr, which is comparable to the 0.5 reversals per Myr we computed from the Ogg (2012) chronology using an interval length of $10 \mathrm{Myr}$. Similarly, the standard deviation 
of the reversal rate of 1000 independent $5 \mathrm{Myr}$ simulations is 0.97 , which is also comparable to the standard deviation of 1.01 reversals per Myr, suggested by the Ogg (2012) chronology, using an interval length of $5 \mathrm{Myr}$.

A candidate for the standard deviation of the timeaveraged VADM is the difference of the time averages of Sint-2000 and PADM2M, which gives $\sigma_{\bar{x}}=0.48 \times$ $10^{22} \mathrm{~A} \mathrm{~m}^{2}$. Similarly, one can define the standard deviation $\sigma_{\sigma}$ by the difference of VADM standard deviations (over time) derived from Sint-2000 and PADM2M. This gives $\sigma_{\sigma}=0.36 \times 10^{22} \mathrm{Am}^{2}$. We can also derive error covariances using the model with nominal parameters and perform 1000 independent $2 \mathrm{Myr}$ simulations. For each simulation, we compute the time average and the VADM standard deviations, which then allows us to compute standard deviations of these quantities. Specifically, we find a value of $0.26 \times 10^{22} \mathrm{~A} \mathrm{~m}^{2}$ for the standard deviation of the time average and $0.11 \times 10^{22} \mathrm{~A} \mathrm{~m}^{2}$ for the standard deviation of the VADM standard deviation. These values are comparable to what we obtained from the data, especially if we base the standard deviations on half of the difference of the PADM2M and Sint-2000 values, i.e., assuming that the data sets are within 2 standard deviations (rather than within 1, which we assumed above).

A difficulty with these candidate error covariances is that we have few time-domain observations compared with the large number of spectral data in the power spectra (see below). This vast difference in the number of time-domain and spectral data means that the spectral data can overwhelm the recovery of model parameters. We address this issue by lowering the error variances $\sigma_{\mathrm{rr}}, \sigma_{\bar{x}}$ and $\sigma_{\sigma}$ by a factor of 100 :

$\sigma_{\text {rr }}=0.05$ reversals per Myr,

$\sigma_{\bar{x}}=0.048 \times 10^{22} \mathrm{Am}^{2}$,

$\sigma_{\sigma}=0.036 \times 10^{22} \mathrm{Am}^{2}$.

Decreasing the error variances of the time-domain data increases the relative importance of the time-domain data compared to the spectral data, which in turn leads to an overall good fit of the model to all data. This comes at the expense of not necessarily realistic posterior error covariances for some or all of the parameters. We discuss these issues in more detail in Sect. 6. Alternatives to the approach we take here (reducing error covariances) include reducing the number of spectral data compared to the number of time-domain data; see also Bärenzung et al. (2018). The difficulty with such an approach, however, is that reducing the number of spectral data is not easy to do and that the consequences such data reduction may have for posterior estimates is difficult to anticipate.

\subsubsection{Low frequencies}

The component $p_{l, \text { lf }}(y \mid \theta)$ of the feature-based likelihood (Eq. 15) addresses the behavior of the dipole at low frequencies of $10^{-4}-0.5$ cycles per kyr and is based on the PSDs of the Sint-2000 and PADM2M data sets. We construct the likelihood using the equation

$y_{\mathrm{lf}}=h_{\mathrm{lf}}(\theta)+\varepsilon_{\mathrm{lf}}$,

where $y_{\mathrm{lf}}$ is a feature that represents the PSD of the Earth's dipole field at low frequencies, where $h_{\mathrm{lf}}(\theta)$ maps the model parameters to the data $y_{\mathrm{lf}}$ and where $\varepsilon_{\mathrm{lf}}$ represents the errors we expect.

We define $y_{\mathrm{lf}}$ as the mean of the PSDs of Sint-2000 and PADM2M. The function $h_{\mathrm{lf}}(\theta)$ maps the model parameters to the feature $y_{\mathrm{lf}}$ and is based on the PSD of the linear model (Eq. 3). To account for the smoothing introduced by sedimentation processes, we define $h_{\mathrm{lf}}(\theta)$ as a function that computes the PSD of the Myr model by using the "unsmoothed" spectrum of Eq. (8) for frequencies less than 0.05 cycles per kyr and uses the "smoothed" spectrum of Eq. (9) for frequencies between 0.05 and 0.5 cycles per kyr:

$$
\begin{aligned}
h_{\mathrm{lf}}(\theta) & =\frac{2 D}{\gamma^{2}+4 \pi^{2} f^{2}} \\
& \cdot \begin{cases}1 & \text { if } f \leq 0.05, \\
\exp \left(-\left(4 \pi^{2} f^{2} T_{\mathrm{s}}^{2}\right) / 12\right) & \text { if } 0.05<f \leq 0.5,\end{cases}
\end{aligned}
$$

Note that $h_{\mathrm{lf}}(\theta)$ does not depend on $\bar{x}$ or $a$. This also means that the data regarding low frequencies are not useful for determining these two parameters (see Sect. 6).

The uncertainty introduced by sampling the VADM once per kyr for only $2 \mathrm{Myr}$ is the dominant source of error in the power spectral densities. For a Gaussian error model $\varepsilon_{\text {lf }}$ with zero mean, this means that the error covariance should describe uncertainties that are induced by the limited amount of data. We construct such a covariance as follows. We perform $10^{4}$ simulations, each of $2 \mathrm{Myr}$, with the nonlinear Myr model (Eq. 4) and its nominal parameters (see Table 1). We compute the PSD of each simulation and build the covariance matrix of the $10^{4}$ PSDs. In Fig. 4a we illustrate the error model by plotting the PSDs of PADM2M (red), Sint-2000 (orange), their mean, $y_{\mathrm{lf}}$ (dark blue), and $5 \times 10^{3}$ samples of $\varepsilon_{\text {lf }}$ added to $y_{\text {lf }}$ (grey).

Since the PSDs of Sint-2000 and PADM2M are well within the cloud of PSDs we generated with the error model, this choice for modeling the expected errors in low-frequency PSDs seems reasonable to us.

For comparison, we also plot $10^{3}$ samples of an error model that only accounts for the reported errors in Sint-2000. This is done by adding independent Gaussian noise, whose standard deviation is given by the Sint-2000 data set every kyr, to the VADM of Sint-2000 and PADM2M. This results in $10^{3}$ "perturbed" versions of Sint-2000 or PADM2M. For each one, we compute the PSD and plot the result in Fig. 4b. The resulting errors are smaller than the errors induced by the shortness of the record. In fact, the reported error does not account for the difference in the Sint-2000 and PADM2M data sets. This suggests that the reported error is too small. 

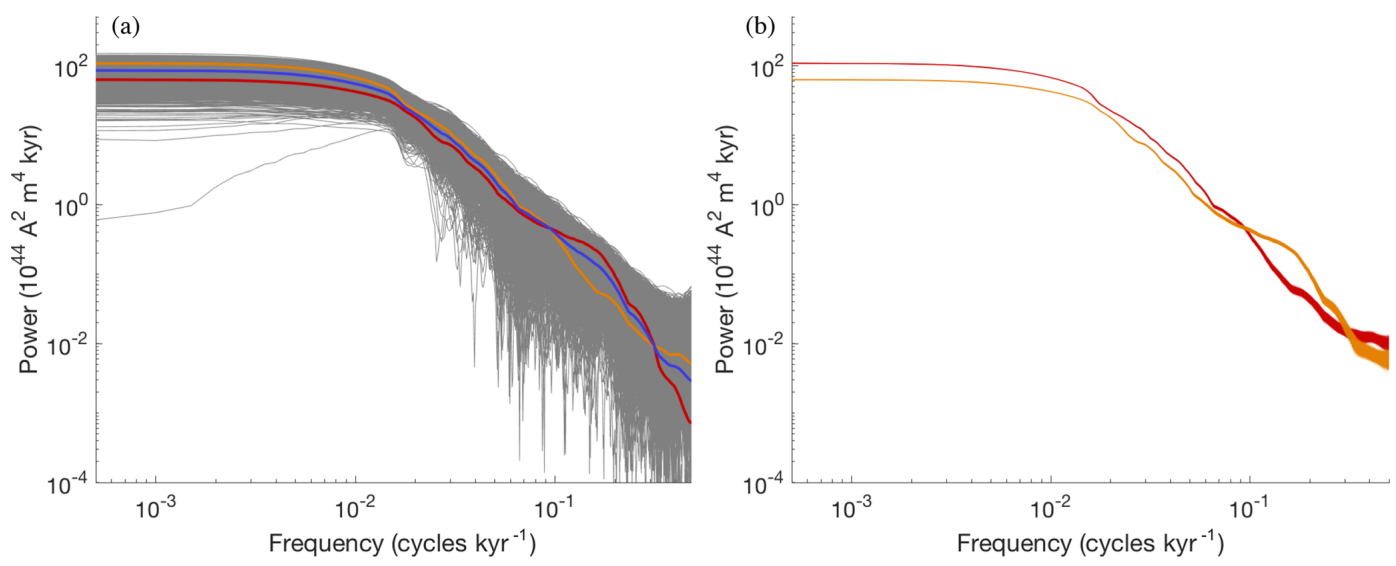

Figure 4. (a) Low-frequency data and error model due to shortness of record. Orange: PSD of Sint-2000. Red: PSD of PADM2M. Blue: mean of PSDs of Sint-2000 and PADM2M ( $\left.y_{\mathrm{lf}}\right)$. Grey: $5 \times 10^{3}$ samples of the error model $\varepsilon_{\mathrm{lf}}$ added to $y_{\mathrm{lf}}$. (b) Error model based on errors in Sint-2000. Orange: $10^{3}$ samples of the PSDs computed from "perturbed" Sint-2000 VADMs. Red: $10^{3}$ samples of the PSDs computed from "perturbed" PADM2M VADMs.

\subsubsection{High frequencies}

We now consider the high-frequency behavior of the model and use the CALS10k.2 data. We focus on frequencies between 0.9 and 9.9 cycles per kyr, where the upper limit is set by the resolution of the CALS10k.2 data. The lower limit is chosen to avoid overlap between the PSDs of CALS10k.2 and Sint-2000/PADM2M. Our choice also acknowledges that the high-frequency part of the PSD for Sint-2000/PADM2M may be less reliable than the PSD of CALS10k.2 for these frequencies. As above, we construct the likelihood $p_{l, \mathrm{hf}}(y \mid \theta)$ from an equation similar to Eq. (23):

$y_{\mathrm{hf}}=h_{\mathrm{hf}}(\theta)+\varepsilon_{\mathrm{hf}}$,

where $y_{\mathrm{hf}}$ is the PSD of CALS10k.2 in the frequency range we consider, where $h_{\mathrm{hf}}(\theta)$ is a function that maps model parameters to the data and where $\varepsilon_{\mathrm{hf}}$ is the error model.

We base $h_{\mathrm{hf}}(\theta)$ on the PSD of the linear model (see Eq. 10) and set

$h_{\mathrm{hf}}(\theta)=\frac{2 D}{\gamma^{2}+4 \pi^{2} f^{2}} \cdot \frac{a^{2}}{a^{2}+4 \pi^{2} f^{2}}$,

where $f$ is the frequency in the range we consider here. Recall that $a^{-1}$ defines the correlation time of the noise in the kyr model.

The error model $\varepsilon_{\mathrm{hf}}$ is Gaussian with mean zero and the covariance is designed to represent errors due to the shortness of the record. This is done, as above, by using $10 \mathrm{kyr}$ simulations of the nonlinear model (Eqs. 6-7) with nominal parameter values. We perform 5000 simulations and for each one compute the PSD over the frequency range we consider (0.9-9.9 cycles per kyr). The covariance matrix computed from these PSDs defines the error model $\varepsilon_{\mathrm{hf}}$, which is illustrated along with the low-frequency error model and the data in Fig. 5.

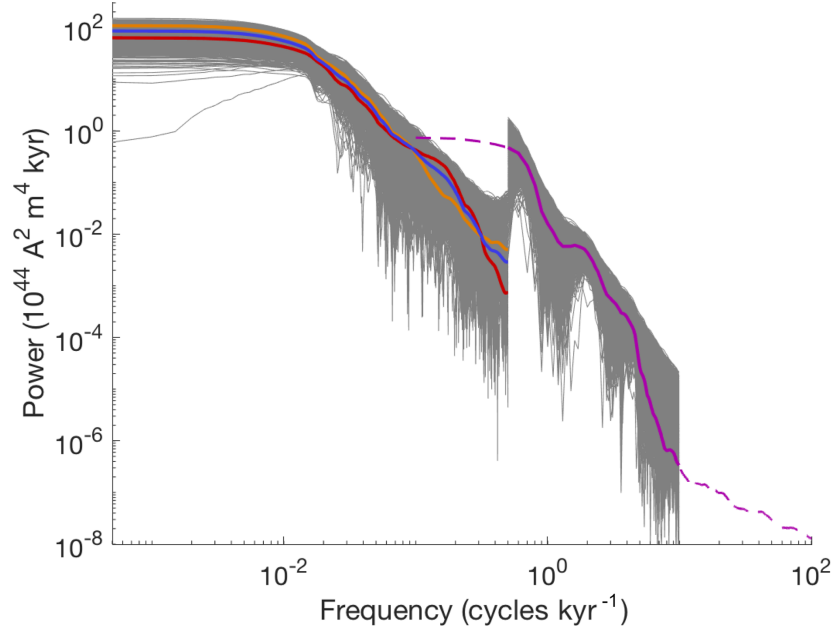

Figure 5. Data and error models for low and high frequencies. Orange: PSD of Sint-2000. Red: PSD of PADM2M. Blue: mean of PSDs of Sint-2000 and PADM2M ( $\left.y_{\mathrm{lf}}\right)$. Grey (low frequencies): $5 \times 10^{3}$ samples of the error model $\varepsilon_{\mathrm{lf}}$ added to $y_{\mathrm{lf}}$. Dashed purple: PSD of CALS10k.2. Solid purple: PSD of CALS10k.2 at frequencies we consider $\left(y_{\mathrm{hf}}\right)$. Grey (high frequencies): $5 \times 10^{3}$ samples of the error model $\varepsilon_{\mathrm{hf}}$ added to $y_{\mathrm{hf}}$.

This concludes the construction of the likelihood and, together with the prior (see Sect. 4.1), we have now formulated the Bayesian formulation of this problem in terms of the posterior distribution (Eq. 14).

\subsection{Numerical solution by MCMC}

We solve the Bayesian parameter estimation problem numerically by Markov chain Monte Carlo (MCMC). This means that we use a "MCMC sampler" that generates samples from the posterior distribution in the sense that averages computed 
over the samples are equal to expected values computed over the posterior distribution in the limit of infinitely many samples. A (Metropolis-Hastings) MCMC sampler works as follows: the sampler proposes a sample by drawing from a proposal distribution and the sample is accepted with a probability to ensure that the stationary distribution of the Markov chain is the targeted posterior distribution.

We use the affine-invariant ensemble sampler, called the MCMC Hammer, of Goodman and Weare (2010), implemented in Matlab by Grinsted (2018). The MCMC Hammer is a general purpose ensemble sampler that is particularly effective if there are strong correlations among the various parameters. The Matlab implementation of the method is easy to use and requires that we provide the sampler with functions that evaluate the prior distribution and the likelihood, as described above.

In addition, the sampler requires that we define an initial ensemble of 10 walkers ( 2 per parameter). This is done as follows. We draw the initial ensemble from a Gaussian whose mean is given by the nominal parameters in Table 3 and whose covariance matrix is a diagonal matrix whose diagonal elements are $50 \%$ of the nominal values. The Gaussian is constrained by the upper and lower bounds in Table 3 . The precise choice of the initial ensemble, however, is not so important as the ensemble generated by the MCMC Hammer quickly spreads out to search the parameter space.

We assess the numerical results by computing integrated autocorrelation time (IACT) using the definitions and methods described by Wolff (2004). The IACT is a measure of how effective the sampler is. We generate an overall number of $10^{6}$ samples, but the number of "effective" samples is $10^{6} / \mathrm{IACT}$. For all MCMC runs we perform (see Sects. 5 and 6), the IACT of the Markov chain is about 100. We discard the first 10 - IACT samples as "burn in", further reducing the impact of the distribution of the initial ensemble. We also ran shorter chains with $10^{5}$ samples and obtained similar results, indicating that the chains of length $10^{6}$ are well resolved.

Recall that all MCMC samplers yield the posterior distribution as their stationary distribution, but the specific choice of MCMC sampler defines "how fast" one approaches the stationary distribution and how effective the sampling is (burn-in time and IACT). In view of the fact that likelihood evaluations are, by our design, computationally inexpensive, we may run (any) MCMC sampler to generate a long chain ( $10^{6}$ samples). Thus, the precise choice of MCMC sampler is not so important for our purposes. We found that the MCMC Hammer solves the problem with sufficient efficiency for our purposes.

The code we wrote is available on github: https://github. com/mattimorzfeld/ (last access: 25 June 2019). It can be used to generate 100000 samples in a few hours and $10^{6}$ samples in less than a day. For this reason, we can run the code in several configurations and with likelihoods that are missing some of the factors that comprise the overall feature- based likelihood (Eq. 15). This allows us to study the impact each individual data set has on the parameter estimates, and it also allows us to assess the validity of some of our modeling choices, in particular with respect to error variances which are notoriously difficult to come by (see Sect. 6).

\section{Results}

We run the MCMC sampler to generate $10^{6}$ samples approximately distributed according to the posterior distribution. We illustrate the posterior distribution by a corner plot in Fig. 6 .

The corner plot shows all one- and two-dimensional histograms of the posterior samples. We observe that the four one-dimensional histograms are well-defined "bumps" whose width is considerably smaller than the assumed parameter bounds (see Table 3) which define the "uninformative", uniform prior. Thus, the posterior probability, which synthesizes the information from the data via the definition of the features, is concentrated over a smaller subset of parameters than the prior probability. In this way, the Bayesian parameter estimation has sharpened the knowledge about the parameters by incorporating the data.

The two-dimensional histograms indicate correlations among the parameters $\theta=\left(\bar{x}, D, \gamma, T_{\mathrm{s}}, a\right)^{T}$, with strong correlations between $\bar{x}, D$ and $\gamma$. These correlations can also be described by the correlation coefficients.

\begin{tabular}{l|rrrrr} 
& $\bar{x}$ & $D$ & $\gamma$ & $T_{\mathrm{S}}$ & $a$ \\
\hline $\bar{x}$ & 1.00 & 0.78 & 0.20 & 0.02 & -0.03 \\
$D$ & 0.78 & 1.00 & 0.64 & 0.02 & -0.03 \\
$\gamma$ & 0.20 & 0.64 & 1.00 & -0.01 & -0.02 \\
$T_{\mathrm{S}}$ & 0.02 & 0.02 & -0.01 & 1.00 & 0.00 \\
$a$ & -0.03 & -0.03 & -0.02 & 0.00 & 1.00
\end{tabular}

The strong correlation between $\bar{x}, D$ and $\gamma$ is due to the contribution of the reversal rate to the overall likelihood (see Eq. 20) and the dependence of the spectral data on $D$ and $\gamma$ (see Eqs. 9 and 10). From the samples, we can also compute means and standard deviations of all five parameters, and we show these values in Table 4.

The table also shows the reversal rate and VADM standard deviation that we compute from 2000 samples of the posterior distribution followed by evaluation of Eqs. (20) and (13) for each sample. We note that the reversal rate (4.06 reversals per Myr) is lower than the reversal rate we used in the likelihood (4.23 reversals per Myr). Since the posterior standard deviation is 0.049 reversals per Myr, the reversal rate data are about 4 standard deviations away from the mean we compute. Similarly, the posterior VADM standard deviation (mean value of $1.77 \times 10^{22} \mathrm{Am}^{2}$ ) is also far, as measured by the posterior standard deviation, from the value we use as data $\left(1.66 \times 10^{22} \mathrm{Am}^{2}\right)$. These large deviations indicate an inconsistency between the VADM standard deviation and the reversal rate. A higher reversal rate could be achieved with a 

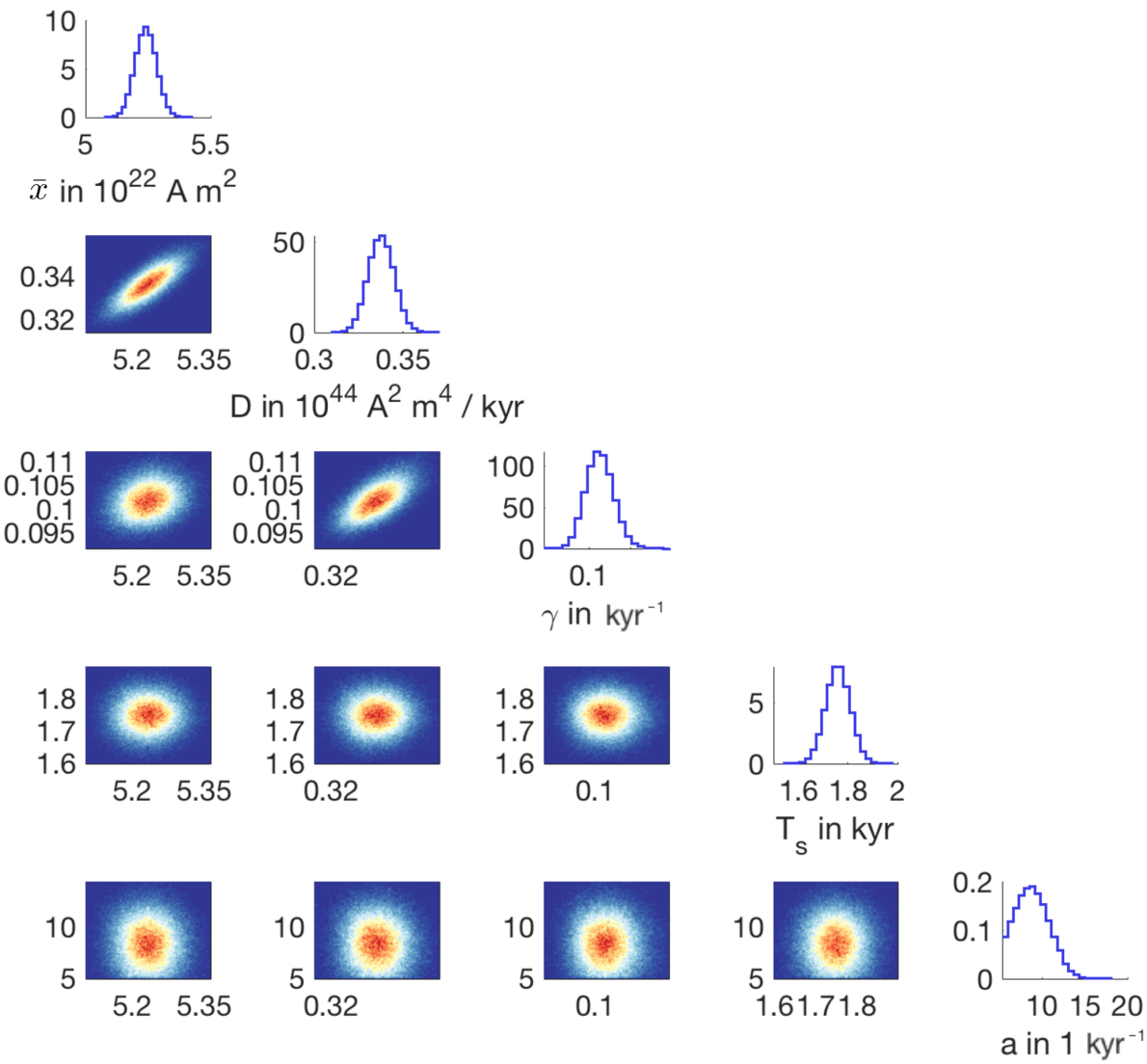

Figure 6. One- and two-dimensional histograms of the posterior distribution.

Table 4. Posterior mean and standard deviation (in brackets) of the model parameters and corresponding estimates of the reversal rate and VADM standard deviation.

\begin{tabular}{lllllll}
\hline $\begin{array}{l}\bar{x} \\
\text { in } 10^{22} \mathrm{Am}^{2}\end{array}$ & $\begin{array}{l}D \\
\text { in } 10^{44} \mathrm{~A}^{2} \mathrm{~m}^{4} \mathrm{kyr}^{-1}\end{array}$ & $\begin{array}{l}\gamma \\
\text { in } \mathrm{kyr}^{-1}\end{array}$ & $\begin{array}{l}T_{\mathrm{S}} \\
\text { in } \mathrm{kyr}\end{array}$ & $\begin{array}{l}a \\
\text { in } \mathrm{kyr}^{-1}\end{array}$ & $\begin{array}{l}\sigma \\
\text { in } 10^{22} \mathrm{Am}^{2}\end{array}$ & $\begin{array}{l}\text { Rev. rate } \\
\text { in reversals per Myr }\end{array}$ \\
\hline $5.23(0.043)$ & $0.34(0.0072)$ & $0.10(0.0033)$ & $1.75(0.050)$ & $8.56(1.93)$ & $1.77(0.024)$ & $4.06(0.049)$ \\
\hline
\end{tabular}

higher VADM standard deviation. The reason is that the reversal rate in Eq. (20) can be re-written as

$r \approx \frac{\gamma}{2 \pi} \cdot \exp \left(-\frac{\bar{x}^{2}}{6 \sigma^{2}}\right) \times 10^{3} \mathrm{Myr}^{-1}$,

using $\sigma \approx \sqrt{D / \gamma}$, i.e., neglecting the correction factor due to sedimentation, which has only a minor effect. Using a time average of $\bar{x}=5.23 \times 10^{22} \mathrm{~A} \mathrm{~m}^{2}$ and a reversal rate of $r=4.2$ reversals per Myr, setting $\gamma=0.1 \mathrm{kyr}^{-1}$ (posterior mean value) and solving for the VADM standard deviation result in $\sigma \approx 1.86 \times 10^{22} \mathrm{~A} \mathrm{~m}^{2}$, which is not compatible with the SINT-2000 and PADM2M data sets (where $\sigma \approx$ $1.66 \times 10^{22} \mathrm{~A} \mathrm{~m}^{2}$ ). One possible source of discrepancy is that the low-frequency data sets underestimate the standard deviation and also the time average. For example, Ziegler et al. (2008) report a time-averaged VADM of $7.64 \times 10^{22} \mathrm{Am}^{2}$ and a standard deviation of $\sigma=2.72 \times 10^{22} \mathrm{~A} \mathrm{~m}^{2}$ for pale- ointensity measurements from the past $0.55 \mathrm{Myr}$. These measurements are unable to provide any constraint on the temporal evolution of the VADM (in contrast to the SINT-2000 and PADM2M models). Instead, these measurements represent a sampling of the steady-state probability distribution for the dipole moment. The results thus suggest that a larger mean and standard deviation are permitted by paleointensity observations. Using the larger values for the time average and VADM standard deviation, but keeping $\gamma=0.1 \mathrm{kyr}^{-1}$ (posterior mean value), leads to a reversal rate of $r \approx 4.27$ reversals per Myr, which is compatible with the reversal rates based on the past $30 \mathrm{Myr}$ in Table 2. It is, however, also possible that the model for the reversal rate has shortcomings. Identifying these shortcomings is a first step in making model improvements and the Bayesian parameter estimation framework we describe is a mathematically and computationally sound tool for discovering such inconsistencies. 
The model fit to the spectral data is illustrated in Fig. 7a.

Here, we plot 100 PSDs, computed from $2 \mathrm{Myr}$ and $10 \mathrm{kyr}$ model runs, and where each model run uses a parameter set drawn at random from the posterior distribution. For comparison, the figure also shows the PADM2M, Sint-2000 and CALS10k. 2 data as well as $5 \times 10^{3}$ realizations of the lowand high-frequency error models. We note that the overall uncertainty is reduced by the Bayesian parameter estimation. The reduction in uncertainty is apparent from the expected errors generating a "wider" cloud of PSDs (in grey) than the posterior estimates (in blue and turquoise). We further note that the PSDs of the models, with parameters drawn from the posterior distribution, fall largely within the expected errors (illustrated in grey). In particular, the high-frequency PSDs (the CALS10k.2 range) are well within the errors we imposed by the likelihoods. The low frequencies of Sint2000/PADM2M are also within the expected errors, and so are the high frequencies beyond the second roll-off due to the sedimentation effects. At intermediate frequencies, some of the PDSs of the model are outside of the expected errors. This indicates a model inconsistency because it is difficult to account for the intermediate frequencies with model parameters that fit the other data (spectral and time domain) within the assumed error models. We investigate this issue further in Sect. 6.

Panels (b) and (c) of Fig. 7 show a Myr model run (top) and kyr model run (bottom) using the posterior mean values for the parameters. We note that the model with posterior mean parameter values exhibits qualitatively similar characteristics to the Sint-2000, PADM2M and CALS10k.2 data. The figure thus illustrates that the feature-based Bayesian parameter estimation, which is based solely on PSD, reversal rates, timeaveraged VADM and VADM standard deviation, translates into model parameters that also appear reasonable when a single simulation in the time domain is considered.

In summary, we conclude that the likelihoods we constructed and the assumptions about errors we made lead to a posterior distribution that constrains the model parameters tightly (as compared to the uniform prior). The posterior distribution describes a set of model parameters that yield model outputs that are comparable with the data in the featurebased sense. The estimates of the uncertainty in the parameters, e.g., posterior standard deviations, however, should be used with the understanding that error variances are not easy to define. For the spectral data, we constructed error models that reflect uncertainty induced by the shortness of the paleomagnetic record. For the time-domain data (reversal rate, time-averaged VADM and VADM standard deviation) we used error variances that are smaller than intuitive error variances to account for the fact that the number of spectral data points (hundreds) is much larger than the number of time-domain data points (three data points). Moreover, the reversal rate and VADM standard deviation data are far (as measured by posterior standard deviations) from the reversal rate and VADM standard deviation of the model with poste- rior parameters. As indicated above, this discrepancy could be due to inconsistencies between spectral data and timedomain data, which we will study in more detail in the next section.

\section{Discussion}

We study the effects the independent data sets have on the parameter estimates and also study the effects of different choices for error variances for the time-domain data (reversal rate, time-averaged VADM and VADM standard deviation). We do so by running the MCMC code in several configurations. Each configuration corresponds to a posterior distribution and, therefore, to a set of parameter estimates. The configurations we consider are summarized in Table 4 and the corresponding parameter estimates are reported in Table 5. Configuration (a) is the default configuration described in the previous sections. We now discuss the other configurations in relation to (a) and in relation to each other.

Configuration (b) differs from configuration (a) in that the CALS10k.2 data are not used; i.e., we do not include the high-frequency component, $p_{l, \mathrm{hf}}(y \mid \theta)$, in the feature-based likelihood (Eq. 15). Configurations (a) and (b) lead to nearly identical posterior distributions and, hence, nearly identical parameter estimates with the exception of the parameter $a$, which controls the correlation of the noise on the kyr timescale. The differences and similarities are apparent when we compare the corner plots of the posterior distributions of configurations (a), shown in Fig. 6, and of configuration (b), shown in Fig. 8.

The corner plots are nearly identical, except for the bottom row of plots, which illustrates marginals of the posterior related to $a$. We note that the posterior distribution over $a$ is nearly identical to its prior distribution. Thus, the parameter $a$ is not constrained by the data used in configuration (b), which is perhaps not surprising because $a$ only appears in the Bayesian parameter estimation problem via the highfrequency likelihood $p_{l, \mathrm{hf}}(y \mid \theta)$. Moreover, since $p_{l, \mathrm{lf}}(y \mid \theta)$ and $p_{l, \mathrm{td}}(y \mid \theta)$ are independent of $a$, the marginal of the posterior distribution of configuration (b) over the parameter $a$ is independent of the data. More interestingly, however, we find that all other model parameters are estimated to have nearly the same values, independently of whether CALS10k.2 is being used during parameter estimation or not. This latter observation indicates that the model is self-consistent and consistent with the data on the Myr and kyr timescales; in the context of our simple stochastic model, the data from CALS10k. 2 mostly constrain the noise correlation parameter $a$.

Configuration (c) differs from configuration (a) in the error variances for the time-domain data (reversal rate, timeaveraged VADM and VADM standard deviation). With the larger values used in configuration (c), the spectral data are emphasized during the Bayesian estimation, which also leads 

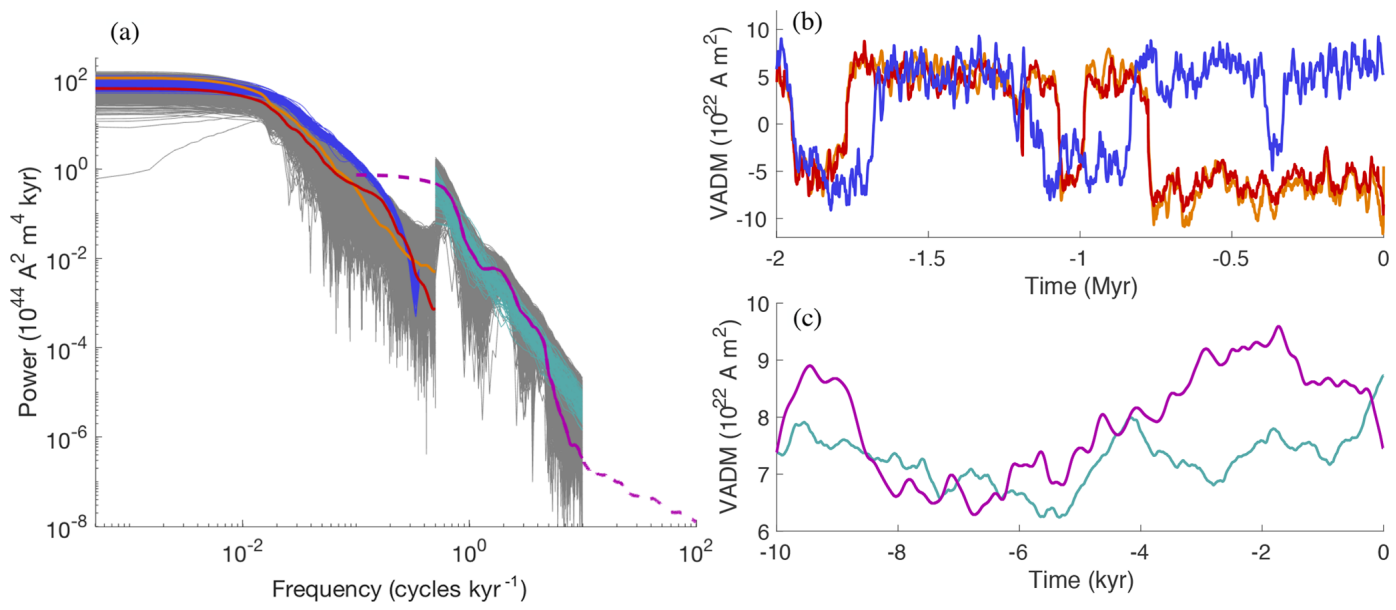

Figure 7. Parameter estimation results. (a) PSDs of data and model. Orange: PSD of Sint-2000. Red: PSD of PADM2M. Dashed purple: PSD of CALS10k.2. Solid purple: PSD of CALS10k.2 at frequencies we consider ( $y_{\mathrm{hf}}$ ). Grey (low frequencies): $5 \times 10^{3}$ samples of the error model $\varepsilon_{\text {lf }}$ added to $y_{\text {lf }}$. Grey (high frequencies): $5 \times 10^{3}$ samples of the error model $\varepsilon_{\mathrm{hf}}$ added to $y_{\mathrm{hf}}$. Dark blue: PSDs of 100 posterior samples of the Myr model (with smoothing). Turquoise: PSDs of 100 posterior samples of the kyr model with uncorrelated noise. (b) Sint-2000 (orange), PADM2M (red) and a realization of the Myr model with smoothing and with posterior mean parameters (blue). (c) CALS10k.2 (purple) and a realization of the kyr model with correlated noise and with posterior mean parameters of the kyr model (turquoise).

Table 5. Configurations for several Bayesian problem formulations. A checkmark means that the data set is used; a cross means it is not used in the overall likelihood construction. The standard deviations $(\sigma)$ define the Gaussian error models for the reversal rate, time-averaged VADM and VADM standard deviation.

\begin{tabular}{lrrrrrr}
\hline Configuration & (a) & (b) & (c) & (d) & (e) & (f) \\
\hline PADM2M \& Sint-2000 & $\checkmark$ & $\checkmark$ & $\checkmark$ & $\checkmark$ & $\times$ & $\checkmark$ \\
CALS10k.2 & $\checkmark$ & $\times$ & $\checkmark$ & $\checkmark$ & $\times$ & $\times$ \\
Rev. Rate, time avg., SD & $\checkmark$ & $\checkmark$ & $\checkmark$ & $\times$ & $\checkmark$ & $\times$ \\
\hline$\sigma_{\text {rr in reversals per Myr }}$ & 0.05 & 0.05 & 0.5 & n/a & 0.05 & n/a \\
$\sigma_{\bar{x}}$ in $10^{22} \mathrm{~A} \mathrm{~m}^{2}$ & 0.048 & 0.048 & 0.48 & n/a & 0.048 & n/a \\
$\sigma_{\sigma}$ in $10^{22} \mathrm{~A} \mathrm{~m}^{2}$ & 0.036 & 0.036 & 0.36 & n/a & 0.036 & n/a \\
\hline
\end{tabular}

n/a: not applicable

to an overall better fit of the spectra. This is illustrated in Fig. 9, where we plot the 100 PSDs generated by 100 (independent) simulations with the model with parameters drawn from the posterior distribution of configuration (c).

For comparison, we also plot the PSDs of PADM2M, Sint2000, CALS10k. 2 and $5 \times 10^{3}$ realizations of the high- and low-frequency error models. In contrast to configuration (a) (see Fig. 7), we find that the PSDs of the model of configuration (c) are all well within the expected errors. On the other hand, the reversal rate drops to about three reversals per Myr, and the time-averaged VADM and VADM standard deviation also decrease significantly as compared to configuration (a). This is caused by the posterior mean of $D$ being decreased by more than $50 \%$, while $\gamma$ and $T_{\mathrm{s}}$ are comparable for configurations (a)-(c). The fact that the improved fit of the PDSs comes at the cost of a poor fit of the reversal rate, time average and standard deviation is another indication of an inconsistency between the reversal rate and the VADM data sets.
As indicated above, one of the strengths of the Bayesian parameter estimation framework we describe here is being able to identify such inconsistencies. Once identified, one can try to fix the model. For example, we can envision a modification of the functional form of the drift term in Eq. (2). A nearly linear dependence of the drift term on $x$ near $x=\bar{x}$ is supported by the VADM data sets, but the behavior near $x=0$ is largely unconstrained. Symmetry of the underlying governing equations suggests that the drift term should vanish, and the functional form adopted in Eq. (2) is just one way that a linear trend can be extrapolated to $x=0$. Other functional forms that lower the barrier between the potential wells would have the effect of increasing the reversal rate. This simple change to the model could bring the reversal rate into better agreement with the time average and standard deviation of the VADM data sets.

In configuration (d), the spectral data are used, but the time-domain data are not used (which corresponds to infi- 
Table 6. Posterior parameter estimates (mean and standard deviation, in brackets) and corresponding VADM standard deviation $(\sigma)$ and reversal rates for five different setups (see Table 5).

\begin{tabular}{lrrr}
\hline Configuration & (a) & (b) & (c) \\
\hline $\bar{x}$ in $10^{22} \mathrm{~A} \mathrm{~m}^{2}$ & $5.23(0.043)$ & $5.23(0.042)$ & $3.56(0.26)$ \\
$D$ in $10^{44} \mathrm{~A}^{2} \mathrm{~m}^{4} \mathrm{kyr}^{-1}$ & $0.34(0.0072)$ & $0.34(0.0072)$ & $0.13(0.014)$ \\
$\gamma$ in kyr & $0.10(0.0033)$ & $0.10(0.0033)$ & $0.081(0.0052)$ \\
$T_{\mathrm{S}}$ in $\mathrm{kyr}^{-1}$ & $1.75(0.050)$ & $1.74(0.050)$ & $1.68(0.14)$ \\
$a$ in $\mathrm{kyr}^{-1}$ & $8.56(1.93)$ & $22.45(10.01)$ & $11.69(3.91)$ \\
\hline$\sigma$ in $10^{22} \mathrm{Am}^{2}$ & $1.77(0.024)$ & $1.77(0.023)$ & $1.22(0.060)$ \\
Rev. rate in reversals per Myr & $4.06(0.049)$ & $4.06(0.023)$ & $3.34(0.52)$ \\
\hline Configuration & $(\mathrm{d})$ & $(\mathrm{e})$ & $(\mathrm{f})$ \\
\hline $\bar{x}$ in $10^{22} \mathrm{Am}^{2}$ & $5.04(2.91)$ & $5.56(0.048)$ & $5.04(2.88)$ \\
$D$ in $10^{44} \mathrm{~A}^{2} \mathrm{~m}^{4} \mathrm{kyr}^{-1}$ & $0.094(0.015)$ & $0.44(0.021)$ & $0.093(0.015)$ \\
$\gamma$ in kyr & $0.078(0.0063)$ & $0.14(0.014)$ & $0.077(0.0064)$ \\
$T_{\mathrm{S}}$ in $\mathrm{kyr}^{-1}$ & $1.64(0.19)$ & $2.98(1.15)$ & $1.64(0.19)$ \\
$a$ in $\mathrm{kyr}^{-1}$ & $12.92(4.79)$ & $22.59(10.08)$ & $22.37(10.13)$ \\
\hline$\sigma$ in $10^{22} \mathrm{Am}^{2}$ & $1.08(0.074)$ & $1.66(0.036)$ & $1.07(0.075)$ \\
Rev. rate in reversals per Myr & $2.89(4.11)$ & $4.23(0.050)$ & $2.83(4.10)$ \\
\hline
\end{tabular}
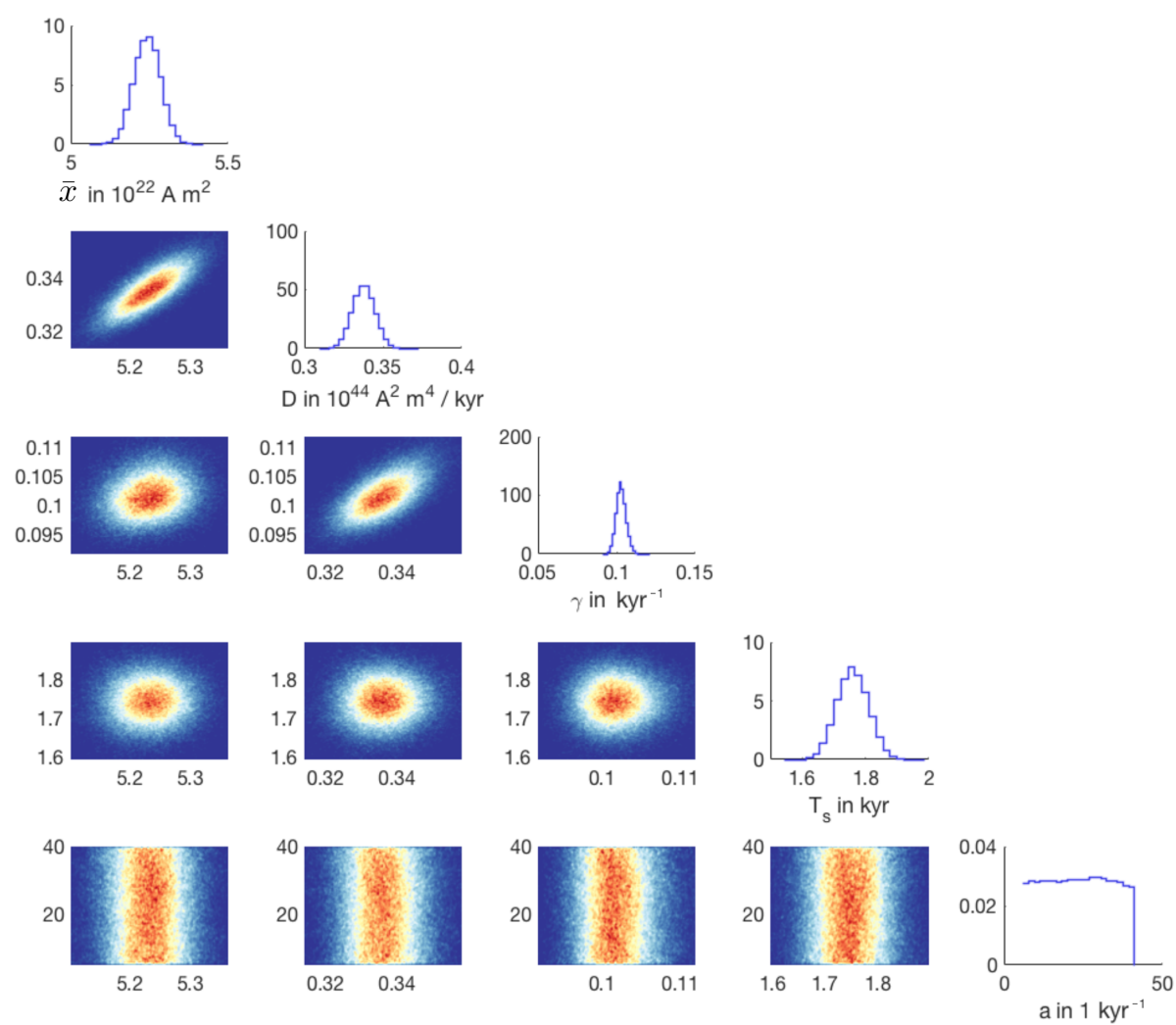

Figure 8. One- and two-dimensional histograms of the posterior distribution of configuration (b). 


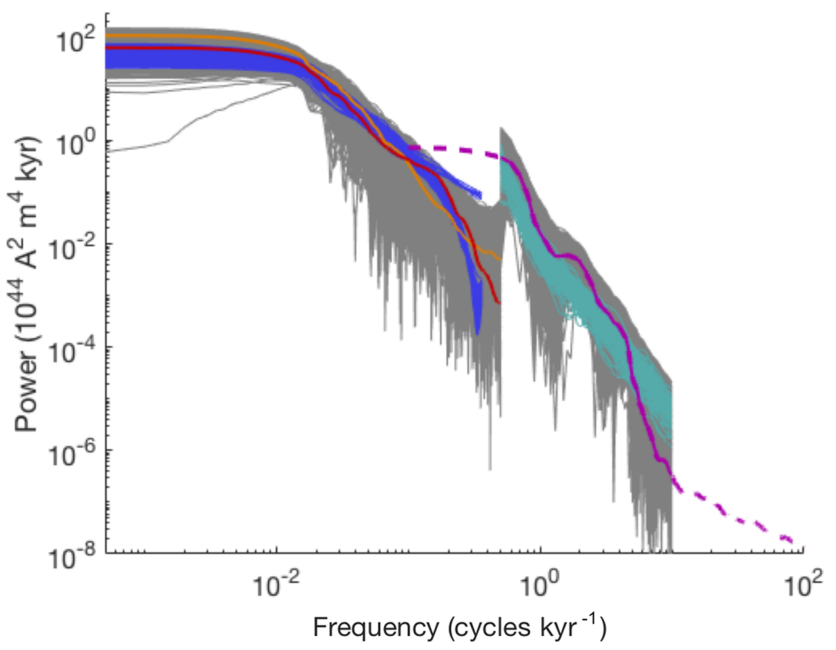

Figure 9. PSDs of data and model with parameters drawn from the posterior distribution of configuration (c). Orange: PSD of Sint2000. Red: PSD of PADM2M. Dashed purple: PSD of CALS10k.2. Solid purple: PSD of CALS10k.2 at frequencies we consider ( $y_{\mathrm{hf}}$ ). Grey (low frequencies): $5 \times 10^{3}$ samples of the error model $\varepsilon_{\mathrm{lf}}$ added to $y_{\mathrm{lf}}$. Grey (high frequencies): $5 \times 10^{3}$ samples of the error model $\varepsilon_{\mathrm{hf}}$ added to $y_{\mathrm{hf}}$. Dark blue: PSDs of 100 posterior samples of the Myr model (with smoothing). Turquoise: PSDs of 100 posterior samples of the kyr model with uncorrelated noise.

nite $\sigma_{\mathrm{rr}}, \sigma_{\sigma}$ and $\sigma_{\bar{x}}$ ). We note that the posterior means and variances of all parameters are comparable for configurations (c), where the error variances of the time-domain data are "large", and (d), where the error variances of the timedomain data are "infinite". Thus, the impact of the timedomain data is minimal if the error variances of the timedomain data are large. The reason is that the number of spectral data points is larger (hundreds) than the number of timedomain data (three data points: reversal rate, time-averaged VADM and VADM standard deviation). When the error variances of the time-domain data decrease, the impact these data have on the parameter estimates increases. We further note that the parameter estimates of configurations (c) or (d) are quite different from the parameter estimates of configuration (a) (see above). For an overall good fit of the model to the spectral and time-domain data, the error variances for the time-domain data must be small, as in configuration (a). Otherwise, the reversal rates are too low. Small error variances, however, imply (relatively) large deviations between the time-domain data and the model predictions. Small error variances also come at the cost of not necessarily realistic posterior variances.

Comparing configurations (d) and (e), we note that if only the spectral data are used, the reversal rates are unrealistically small (nominally one reversal per Myr). Moreover, the parameter estimates based on the spectral data are quite different from the estimates we obtain when we use the timedomain data (reversal rate, time-averaged VADM and VADM standard deviation). This is further evidence that either the model has some inconsistencies or that the reversal rate and the VADM standard deviation are not consistent. Specifically, our experiments suggest that a good match to spectral data requires a set of model parameters that are quite different from the set of model parameters that lead to a good fit to the reversal rate, time-averaged VADM and VADM standard deviation. Experimenting with different functional forms for the drift term is one strategy for achieving better agreement between the reversal rate, the time-averaged VADM and VADM standard deviation.

Comparing configurations (d) and (f), we can further study the effects that the CALS10k.2 data have on parameter estimates (similarly to how we compared configurations (a) and (b) above). The results, shown in Table 6, indicate that the parameter estimates based on configurations (d) and (f) are nearly identical, except in the parameter $a$ that controls the time correlation of the noise on the kyr timescale. This confirms what we already found by comparing configurations (a) and (b): the CALS10k.2 data are mostly useful for constraining $a$. These results, along with configurations (a) and (b), suggest that the model is self-consistent with the independent data on the Myr scale (Sint-2000 and PADM2M) and on the kyr scale (CALS10k.2). Our experiments, however, also suggest that the model has difficulties in reconciling the spectral and time-domain data.

Finally, note that the data used in configuration (d) do not inform the parameter $\bar{x}$, and configuration (f) does not inform $\bar{x}$ or $a$. If the data do not inform the parameters, then the posterior distribution over these parameters is essentially equal to the prior distribution, which is uniform. This is illustrated in Fig. 10, where we show the corner plot of the posterior distribution of configuration (f).

We can clearly identify the uniform prior in the marginals over the parameters $\bar{x}$ and $a$. This means that the Sint-2000 and PADM2M data only constrain the parameters $D, \gamma$ and $T_{\mathrm{s}}$.

\section{Examples of applications of the model}

The Bayesian estimation technique we describe leads to a model with stochastic parameters whose distributions are informed by the paleomagnetic data. Moreover, we ran a large number of numerical experiments to understand the limitations of the model, to discover inconsistencies between the model and the data and to check our assumptions about error modeling. This process results in a well-understood and wellfounded stochastic model for selected aspects of the longterm behavior of the geomagnetic dipole field. We believe that such a model can be useful for a variety of purposes, including testing hypotheses about selected long-term aspects of the geomagnetic dipole.

For example, it was noted by Ziegler et al. (2011) that the VADM (time-averaged) amplitude during the past chron 

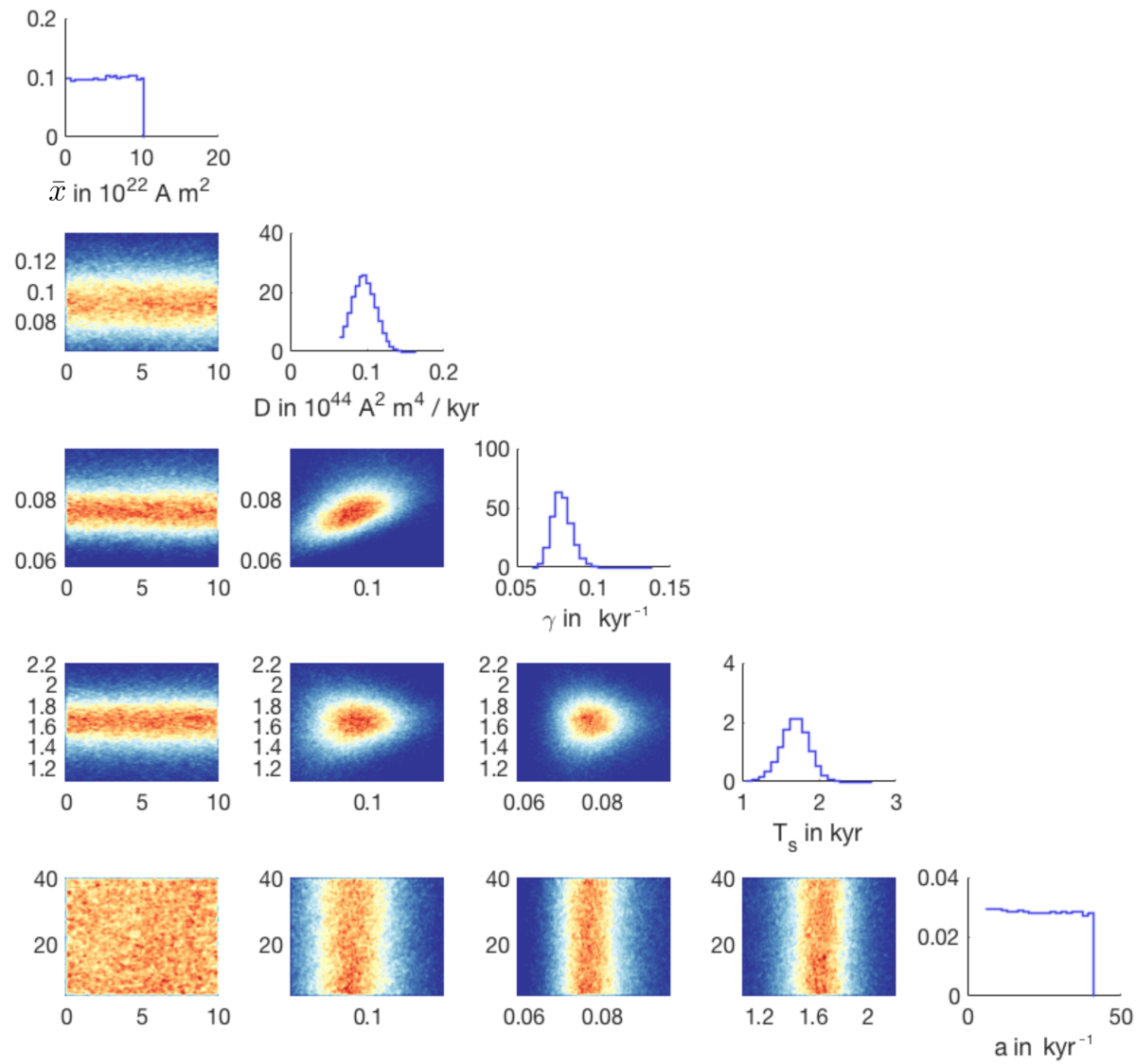

Figure 10. One- and two-dimensional histograms of the posterior distribution of configuration (f).

was slightly lower than during the previous chron. Specifically, the time-averaged VADM for $-0.78<t<0 \mathrm{Myr}$ is $E(x)=6.2 \times 10^{22} \mathrm{Am}^{2}$, but for $-2<t<-0.78 \mathrm{Myr}$ the time average is $E(x)=4.8 \times 10^{22} \mathrm{Am}^{2}$. A natural question is whether this increase in the time average is significant or whether it is due to random variability. We investigate this question using the model whose parameters are the posterior mean values of configuration (a) (the configuration that leads to an overall good fit to all data). Specifically, we perform 10000 simulations of duration $0.78 \mathrm{Myr}$ and 10000 independent simulations of duration 1.22 Myr. For each simulation, we compute the time average, which allows us to estimate the standard deviation of the difference in means (assuming no correlation between the two time intervals). We find that this standard deviation is about $0.46 \times 10^{22} \mathrm{~A} \mathrm{~m}^{2}$, which is much smaller than the differences in VADM time averages of $1.4 \times 10^{22} \mathrm{Am}^{2}$. This suggests that the increase in timeaveraged VADM is likely not due to random variability.

A similar approach can be applied to the question of changes in the reversal rate over geological time. The observed reversal rate over the past $30 \mathrm{Myr}$ is approximately 4.26 $\mathrm{Myr}^{-1}$. When the record is divided into $10 \mathrm{Myr}$ intervals, the reversal rate varies about the average, with a standard deviation of about $0.49 \mathrm{Myr}^{-1}$ (see Table 2). These vari- ations are within the expected fluctuations for the stochastic model. Specifically, we can use an ensemble of $10^{5}$ simulations, each of duration $10 \mathrm{Myr}$, to compute the average and standard deviations of the reversal rate. The results, obtained by using nominal parameters and posterior mean parameters of configurations (a) (overall good fit to all data) and (e) (emphasis on reversal rate data), are shown in Table 7.

As already indicated in Sect. 4.2.1, the standard deviation from the geomagnetic polarity timescale is comparable to the standard deviation we compute via the model. The observed reversal rate for the $10 \mathrm{Myr}$ interval between 30 and $40 \mathrm{Myr}$, however, is approximately $2.0 \mathrm{Myr}^{-1}$, which departs from the $0-30$ Myr average by more than 3 standard deviations. This suggests that the reversal rate between 30 and $40 \mathrm{Ma}$ cannot be explained by natural variability in the model. Instead, it suggests that model parameters were different before $30 \mathrm{Ma}$, implying that there was a change in the operation of the geodynamo.

\section{Summary and conclusions}

We considered parameter estimation for a model of Earth's axial magnetic dipole field. The idea is to estimate the model parameters using data that describe Earth's dipole field over 
Table 7. Average reversal rate and standard deviation of an ensemble of $10^{5}$ simulations of duration $10 \mathrm{Myr}$, filtered to a resolution of $30 \mathrm{kyr}$. The simulations are done with nominal parameter values (Table 3) or the posterior mean values of configurations (a) and (e).

\begin{tabular}{lrrr}
\hline & Nominal parameter values & Configuration (a) & Configuration (e) \\
\hline Average reversal rate $\left(\mathrm{in} \mathrm{Myr}^{-1}\right.$ ) & 4.50 & 3.76 & 3.56 \\
Standard deviation (in $\mathrm{Myr}^{-1}$ ) & 0.68 & 0.63 & 0.59 \\
\hline
\end{tabular}

kyr and Myr timescales. The resulting model, with calibrated parameters, is thus a representation of Earth's dipole field on these timescales. We formulated a Bayesian estimation problem in terms of "features" that we derived from the model and data. The data include two time series (Sint-2000 and PADM2M) that describe the strengths of Earth's dipole over the past $2 \mathrm{Myr}$, a shorter record (CALS10k.2) that describes dipole strength over the past $10 \mathrm{kyr}$, as well as reversal rates derived from the geomagnetic polarity timescale. The features are used to synthesize information from these data sources (that had previously been treated separately).

Formulating the Bayesian estimation problem requires definition of anticipated model error. We found that the main source of uncertainty is the shortness of the paleomagnetic record and constructed error models to incorporate this uncertainty. Numerical solution of the feature-based estimation problem is done via conventional Markov chain Monte Carlo (an affine-invariant ensemble sampler). With suitable error models, our numerical results indicate that the paleomagnetic data constrain all model parameters in the sense that the posterior probability mass is concentrated on a smaller subset of parameters than the prior probability. Moreover, the posterior parameter values yield model outputs that fit the data in a precise, feature-based sense, which also translates into a good fit by other, more intuitive measures.

A main advantage of our approach (Bayesian estimation with an MCMC solution) is that it allows us to understand the limitations and remaining (posterior) uncertainties of the model. After parameter estimation, we thus have produced a reliable, stochastic model for selected aspects of the longterm behavior of the geomagnetic dipole field whose limitations and errors are well-understood. We believe that such a model is useful for hypothesis testing and have given several examples of how the model can be used in this context. Another important aspect of our overall approach is that it can reveal inconsistencies between model and data. For example, we ran a suite of numerical experiments to assess the internal consistency of the data and the underlying model. We found that the model is self-consistent on the Myr and kyr timescales, but we discovered inconsistencies that make it difficult to achieve a good fit to all data simultaneously. It is also possible that the data themselves are not entirely self-consistent in this regard. Our methodology does not resolve these questions, but once inconsistencies are identified, several strategies can be pursued to resolve them, e.g., improving the model or resolving consistency issues of the data themselves. Our conceptual and numerical framework can also be used to reveal the impact that some of the individual data sets have on parameter estimates and associated posterior uncertainties. In this paper, however, we focused on describing the mathematical and numerical framework and only briefly mention some of the implications.

Code and data availability. The code and data used in this paper are available on github: https://github.com/mattimorzfeld (last access: 25 June 2019).

Author contributions. $\mathrm{MM}$ and $\mathrm{BAB}$ performed the research and wrote the paper and accompanying code.

Competing interests. The authors declare that they have no conflict of interest.

Acknowledgements. We thank Johannes Wicht of the Max Planck Institute for Solar System Research and an anonymous reviewer for careful comments that helped in improving our paper. We also thank Andrew Tangborn of NASA GSFC for interesting discussion and useful comments.

Financial support. Matthias Morzfeld has been supported by the National Science Foundation (grant no. DMS-1619630) and by the Alfred P. Sloan Foundation. Bruce A. Buffett has been supported by the National Science Foundation (grant no. EAR-164464).

Review statement. This paper was edited by Jörg Büchner and reviewed by Johannes Wicht and two anonymous referees.

\section{References}

Asch, M., Bocquet, M., and Nodet, M.: Data assimilation: methods, algorithms and applications, SIAM, Philadelphia, 2017.

Bärenzung, J., Holschneider, M., Wicht, J., Sanchez, S., and Lesur, V.: Modeling and Predicting the Short-Term Evolution of the Geomagnetic Field, J. Geophys. Res.-Sol. Ea., 123, 4539-4560, https://doi.org/10.1029/2017JB015115, 2018.

Buffett, B.: Dipole fluctuations and the duration of geomagnetic polarity transitions, Geophys. Res. Lett., 42, 7444-7451, 2015. 
Buffett, B. and Davis, W.: A Probabilistic Assessment of the Next Geomagnetic Reversal, Geophys. Res. Lett., 45, 1845-1850, https://doi.org/10.1002/2018GL077061, 2018.

Buffett, B. and Matsui, H.: A power spectrum for the geomagnetic dipole moment, Earth Planet. Sc. Lett., 411, 20-26, 2015.

Buffett, B. and Puranam, A.: Constructing stochastic models for dipole fluctuations from paleomagnetic observations, Phys. Earth Planet. In., 272, 68-77, 2017.

Buffett, B., Ziegler, L., and Constable, C.: A stochastic model for paleomagnetic field variations, Geophys. J. Int., 195, 86-97, 2013.

Buffett, B. A., King, E. M., and Matsui, H.: A physical interpretation of stochastic models for fluctuations in the Earth's dipole field, Geophys. J. Int., 198, 597-608, 2014.

Cande, S. and Kent, D.: Revised calibration of the geomagnetic polarity timescale for the late Cretaceous and Cenozoic, J. Geophys. Res.-Sol. Ea., 100, 6093-6095, 1995.

Chorin, A. and Hald, O.: Stochastic tools in mathematics and science, Springer, New York, third edn., 2013.

Constable, C. and Johnson, C.: A paleomagnetic power spectrum, Phys. Earth Planet. In., 153, 61-73, 2005.

Constable, C., Korte, M., and Panovska, S.: Persistent high paleosecular variation activity in southern hemisphere for at least 10000 years, Earth Planet. Sc. Lett., 453, 78-86, 2016.

Finlay, C., Olsen, N., Kotsiaros, S., Gillet, N., and Lars, T.: Recent geomagnetic secular variation from Swarm and ground observatories as estimated in the CHAOS-6 geomagnetic field model, Earth Planets Space, 68, 1-18, 2016.

Gissinger, C.: A new deterministic model for chaotic reversals, The European Phys. J. B, 85, 137, https://doi.org/10.1140/epjb/e2012-20799-5, 2012.

Goodman, J. and Weare, J.: Ensemble samplers with affine invariance, Comm. App. Math. Com. Sc., 5, 65-80, 2010.

Grinsted, A.: GWMCMC, https://github.com/grinsted/ (last access: 25 June 2019), 2018.

Hoyng, P., Ossendrijver, M., and Schmitt, D.: The geodynamo as a bistable oscillator, Geophys. Astro. Fluid, 94, 263-314, 2001.

Hoyng, P., Schmitt, D., and Ossendrijver, M.: A theoretical analysis of the observed variability of the geomagnetic dipole field, Phys. Earth Planet. In., 130, 143-157, 2002.

Hulot, G., Finlay, C. C., Constable, C. G., Olsen, N., and Mandea, M.: The magnetic field of planet Earth, Space Sci. Rev., 152, 159-222, 2010.

Lowrie, W. and Kent, D.: Geomagnetic polarity time scale and reversal frequency regimes, Geoph. Monog. Series, 145, 117-129, 2004.

Maclean, J., Santitissadeekorn, N., and Jones, C. K.: A coherent structure approach for parameter estimation in Lagrangian Data Assimilation, Physica D, 360, 36-45, https://doi.org/10.1016/j.physd.2017.08.007, 2017.

Meduri, D. and Wicht, J.: A simple stochastic model for dipole moment fluctuations in numerical dynamo simulations, Frontiers Earth Sci., 4, https://doi.org/10.3389/feart.2016.00038, 2016.
Morzfeld, M., Fournier, A., and Hulot, G.: Coarse predictions of dipole reversals by low-dimensional modeling and data assimilation, Phys. Earth Planet. In., 262, 8-27, https://doi.org/10.1016/j.pepi.2016.10.007, 2017.

Morzfeld, M., Adams, J., Lunderman, S., and Orozco, R.: Featurebased data assimilation in geophysics, Nonlin. Processes Geophys., 25, 355-374, https://doi.org/10.5194/npg-25-355-2018, 2018.

Ogg, J.: Geomagnetic polarity time scale, in: The geological time scale 2012, edited by: Gradstein, F., Ogg, J., Schmitz, M., and Ogg, G., chap. 5, pp. 85-1130, Elsevier Science, Boston, 2012.

Pétrélis, F. and Fauve, S.: Chaotic dynamics of the magnetic field generated by dynamo action in a turbulent flow, J. Phys.Condensed Matter, 20, 494203, https://doi.org/10.1088/09538984/20/49/494203, 2008.

Pétrélis, F., Fauve, S., Dormy, E., and Valet, J.-P.: Simple mechanism for reversals of Earth's magnetic field, Phys. Rev. Lett., 102, 144503, https://doi.org/10.1103/PhysRevLett.102.144503, 2009.

Pourovskii, L., Mravlje, J., Georges, A., Simak, S., and Abrikosov, I.: Electron-electron scattering and thermal conductivity of epsilon-iron at Earth's core conditions, New J. Phys., 19, 073022, https://doi.org/10.1088/1367-2630/aa76c9, 2017.

Reich, S. and Cotter, C.: Probabilistic Forecasting and Bayesian Data Assimilation, Cambridge University Press, Cambridge, 2015.

Rikitake, T.: Oscillations of a system of disk dynamos, Math. Proc. Cambridge, 54, 89-105, 1958.

Risken, H.: The Fokker-Planck equation: Methods of solution and applications, Springer, Berlin, 1996.

Roberts, A. and Winkhofer, M.: Why are geomagnetic excursions not always recorded in sediments? Constraints from postdepositional remanent magnetization lock-in modeling, Earth Planet. Sc. Lett., 227, 345-359, 2004.

Schmitt, D., Ossendrijver, M., and Hoyng, P.: Magnetic field reversals and secular variation in a bistable geodynamo model, Phys. Earth Planet. In., 125, 119-124, 2001.

Valet, J.-P., Meynadier, L., and Guyodo, Y.: Geomagnetic field strength and reversal rate over the past 2 million years, Nature, 435, 802-805, 2005.

Wolff, U.: Monte Carlo errors with less errors, Comput. Phys. Commun., 156, 143-153, 2004.

Ziegler, L., Constable, C., and Johnson, C.: Testing the robustness and limitations of $0-1 \mathrm{Ma}$ absolute paleointensity data, Phys. Earth Planet. In., 170, 34-45, 2008.

Ziegler, L. B., Constable, C. G., Johnson, C. L., and Tauxe, L.: PADM2M: a penalized maximum likelihood model of the 0 2 Ma paleomagnetic axial dipole model, Geophys. J. Int., 184, 1069-1089, 2011. 